\title{
Insulin receptor substrates differentially exacerbate insulin-mediated left ventricular remodeling
}

Christian Riehle, ${ }^{1,2,3,4,5}$ Eric T. Weatherford, ${ }^{1,2}$ Adam R. Wende, ${ }^{3,4,6}$ Bharat P. Jaishy, ${ }^{1,2,3,4}$ Alec W. Seei, ${ }^{1,2}$ Nicholas S. McCarty, ${ }^{1,2}$ Monika Rech, ${ }^{3,4}$ Qian Shi, ${ }^{1,2,7}$ Copireddy R. Reddy, ${ }^{7}$ William J. Kutschke, ${ }^{8}$ Karen Oliveira, ${ }^{3,4}$ Karla Maria Pires, ${ }^{3,4}$ Joshua C. Anderson, ${ }^{9}$ Nikolaos A. Diakos, ${ }^{4}$ Robert M. Weiss, ${ }^{8}$ Morris F. White, ${ }^{10}$ Stavros C. Drakos, ${ }^{11}$ Yang K. Xiang, ${ }^{7,12}$ and E. Dale Abel $\left.\right|^{1,2,3,4}$

1Fraternal Order of Eagles Diabetes Research Center and 2Division of Endocrinology and Metabolism, Roy J. and Lucille A. Carver College of Medicine, University of lowa, lowa City, lowa, USA. ${ }^{3}$ Division of Endocrinology, Metabolism and Diabetes, and ${ }^{4}$ Program in Molecular Medicine, University of Utah School of Medicine, Salt Lake City, Utah, USA. ${ }^{5}$ Department of Cardiology and Angiology, Hannover Medical School, Hannover, Germany. ${ }^{6}$ Division of Molecular and Cellular Pathology, Department of Pathology, University of Alabama at Birmingham, Birmingham, Alabama, USA. 'Department of Pharmacology, UCD, Davis, California, USA. ${ }^{8}$ Division of Cardiovascular Medicine, Department of Internal Medicine, Roy J. and Lucille A. Carver College of Medicine, University of lowa, lowa City, lowa, USA. 'Department of Radiation Oncology, School of Medicine, University of Alabama at Birmingham, Birmingham, Alabama, USA. ${ }^{10}$ Division of Endocrinology, Boston Children's Hospital, Boston, Massachusetts, USA. "'Nora Eccles Harrison Cardiovascular Research and Training Institute (CVRTI), University of Utah School of Medicine, Salt Lake City, Utah, USA. ${ }^{12}$ VA Northern California Health Care System, Mather, California, USA.

Pressure overload (PO) cardiac hypertrophy and heart failure are associated with generalized insulin resistance and hyperinsulinemia, which may exacerbate left ventricular (LV) remodeling. While PO activates insulin receptor tyrosine kinase activity that is transduced by insulin receptor substrate 1 (IRS1), the present study tested the hypothesis that IRS1 and IRS2 have divergent effects on $P O$-induced LV remodeling. We therefore subjected mice with cardiomyocyte-restricted deficiency of IRS1 (CIRS1KO) or IRS2 (CIRS2KO) to PO induced by transverse aortic constriction (TAC). In WT mice, TAC-induced LV hypertrophy was associated with hyperactivation of IRS1 and Akt1, but not IRS2 and Akt2. CIRS1KO hearts were resistant to cardiac hypertrophy and heart failure in concert with attenuated Akt1 activation. In contrast, CIRS2KO hearts following TAC developed more severe LV dysfunction than WT controls, and this was prevented by haploinsufficiency of Akt1. Failing human hearts exhibited isoform-specific IRS1 and Akt1 activation, while IRS2 and Akt2 activation were unchanged. Kinomic profiling identified IRS1 as a potential regulator of cardioprotective protein kinase G-mediated signaling. In addition, gene expression profiling revealed that IRS1 signaling may promote a proinflammatory response following PO. Together, these data identify IRS1 and Akt1 as critical signaling nodes that mediate LV remodeling in both mice and humans.

Authorship note: CR and ETW contributed equally to this work.

Conflict of interest: The authors have declared that no conflict of interest exists.

Copyright: (c) 2020, American Society for Clinical Investigation.

Submitted: November 13, 2019 Accepted: February 26, 2020 Published: March 26, 2020.

Reference information: JCI Insight. 2020;5(6):e134920. https://doi.org/10.1172/jci. insight.134920.

\section{Introduction}

Heart failure is a leading cause of death worldwide. The associated prognosis is worse than prognoses for many malignancies, with about half of patients dying within 5 years of the initial diagnosis (1). Peripheral insulin resistance is a common characteristic of heart failure and type 2 diabetes (T2D) that results in initial compensatory hyperinsulinemia and increased proximal myocardial insulin signaling $(2,3)$. Numerous epidemiologic studies indicate that insulin resistance and diabetes are risk factors for heart failure, of ischemic and nonischemic origins (3). Patients with T2D are commonly treated with insulin in later stages of the disease, and hyperinsulinemia has been associated with impaired myocardial contractile function in humans and animal studies $(4,5)$. Moreover, tight metabolic control of type 2 diabetics achieved by intensifying insulin treatment and the use of certain insulin secretagogues have been associated with adverse 
cardiovascular outcomes - such as increased myocardial infarctions and increased mortality (6) - despite decreased microvascular complications, including diabetic nephropathy and neuropathy (7).

Myocardial insulin signaling is transduced by insulin receptor substrates 1 (IRS1) and IRS2. Previous studies utilizing mice with germline deletion of IRS proteins suggest a predominant role for IRS1 in regulating somatic growth and for IRS2 in the regulation of metabolism (8-10). Using a mouse model, we reported that pressure overload (PO) hypertrophy induced by transverse aortic constriction (TAC) resulted in activated cardiac IR-mediated signaling in the presence of hepatic insulin resistance. Importantly, that same study showed reducing cardiomyocyte insulin signaling attenuated the onset of heart failure under conditions of PO (11). However, the underlying mechanisms remain incompletely understood. We sought to explore the signaling mechanisms responsible for this phenomenon and to determine the respective contribution of IRS isoforms. Therefore, mice with cardiomyocyte-specific deletion of IRS1 (CIRS1KO) or IRS2 (CIRS2KO) were subjected to PO induced by TAC surgery. Our results revealed a critical role for IRS1 - but not for IRS2 - in exacerbating heart failure, and they identify some molecular mechanisms that might link insulin signaling, PO, and heart failure.

\section{Results}

IRS1, but not IRS2, is activated under PO conditions. To test whether IRS isoforms play divergent roles in the adaptations to PO, we determined their protein levels and activation status as indicated by tyrosine phosphorylation 4 weeks after TAC in WT hearts. Interestingly, total IRS1 protein levels increased by $63.6 \%$ $(P<0.05)$, while IRS2 protein levels were unchanged relative to Sham-operated controls after TAC (Figure 1, A and B). IRS1 tyrosine phosphorylation normalized to total IRS1 content increased by about 3-fold $(P<0.05)$ after TAC surgery relative to Sham controls. In contrast, IRS2 tyrosine phosphorylation normalized to IRS2 content was not altered by TAC surgery (Figure 1C). Together, these data indicate that IRS1, but not IRS2, is activated under PO conditions.

IRS1 deficiency protects against LV remodeling and heart failure in response to TAC. We previously reported activation of the IR/Akt signaling cascade under PO conditions (11). Based on our observation that IRS1 is activated in an isoform-specific manner under PO conditions, we hypothesized that IRS1, but not IRS2, mediates PO-induced activation of the Akt/mTOR signaling pathway to induce adverse LV remodeling. We therefore subjected CIRS1KO and CIRS2KO mice to TAC surgery. Contractile function, as measured by transthoracic echocardiography, was decreased in all 3 genotypes 2 days after surgery, as determined by ejection fraction (WT, $-24.7 \%$; CIRS1KO, $-51.3 \%$; CIRS2KO, $-53.6 \% ; P<0.05$ each). Importantly, CIRS1KO mice completely recovered by 2 weeks after TAC, while contractile function remained impaired in WT and CIRS2KO hearts at this time point (WT, $-17.4 \%$; CIRS2KO, $-45.2 \% ; P<0.05$ each; Figure $1, \mathrm{D}$ and $\mathrm{E}$ and Supplemental Table 1 and Supplemental Table 2; supplemental material available online with this article; https://doi.org/10.1172/jci.insight.134920DS1). Similarly, LV dilation as determined by LV end-systolic area at systole was observed in CIRS1KO mice 2 days, but not 2 weeks, after TAC (Figure 1, F and G). Four weeks after TAC, WT hearts displayed cardiac hypertrophy with heart weight/tibia length ratio (HW/ TL) increased relative to Sham surgery ( $+54.4 \%$ vs. Sham, $P<0.05$; Figure $1, \mathrm{H}-\mathrm{J}$ and Supplemental Table 3). This hypertrophic response was attenuated in CIRS1KO hearts (HW/TL, $+18.6 \%$ vs. Sham, $P<0.05$ ) and was exacerbated in CIRS2KO hearts $(\mathrm{HW} / \mathrm{TL},+67.4 \%$ vs. Sham, $P<0.05)$. Stereological quantification revealed decreased cardiomyocyte nuclei number per area in WT and CIRS2KO hearts $(-38.8 \%$ and $-32.1 \%$, respectively; $P<0.05)$ but not in CIRS1KO hearts following TAC surgery. Mean cross-sectional area of cardiomyocytes increased in WT and CIRS2KO hearts following TAC surgery $(+75.8 \%$ and $+80.9 \%$, respectively; $P<0.05$ ) but did not change in CIRS1KO hearts following TAC. Similarly, interstitial tissue volume (an estimate of fibrosis) was increased in WT and CIRS2KO hearts $(+45.9 \%$ and $+60.6 \%$, respectively; $P<0.05$ ) but not in CIRS1KO hearts under PO conditions (Figure 1, K-O). Maximal rates of increase (Max dP/dt) and decrease (Min $\mathrm{dP} / \mathrm{dt}$ ) in LV pressure in vivo were decreased in WT hearts $(-19.3 \%$ and $-16.1 \%$, respectively) but were unchanged in TAC-operated CIRS1KO mice relative to their Sham controls. However, Max $\mathrm{dP} / \mathrm{dt}$ and Min $\mathrm{dP} / \mathrm{dt}$ were impaired to a greater extent by TAC surgery in CIRS2KO hearts $(-38.3 \%$ and $-25.2 \%$, respectively, vs. CIRS2KO Sham, $P<0.05$; Figure $1, \mathrm{P}$ and $\mathrm{Q}$, and Supplemental Table 4). Together, these data indicate that signaling mediated by IRS1, but not IRS2, contributes to adverse LV remodeling under PO conditions.

Deletion of IRS1 prevents activation of Akt1/mTOR signaling in response to PO. To determine the underlying mechanism responsible for the decreased hypertrophic response observed in CIRS1KO hearts, we investigated 
A
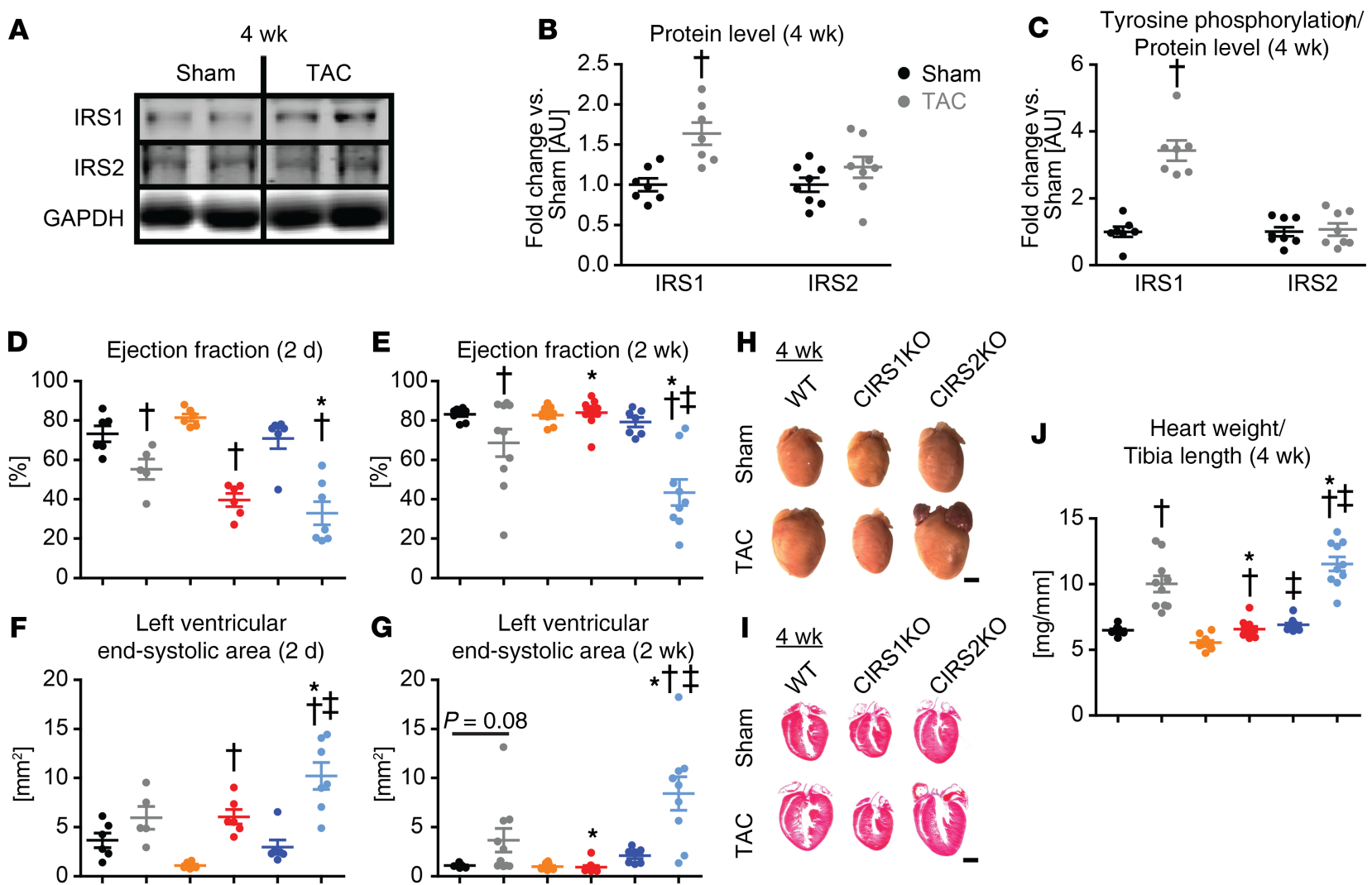

K $\underline{4 w k}$ WT

CIRS1KO

CIRS2KO
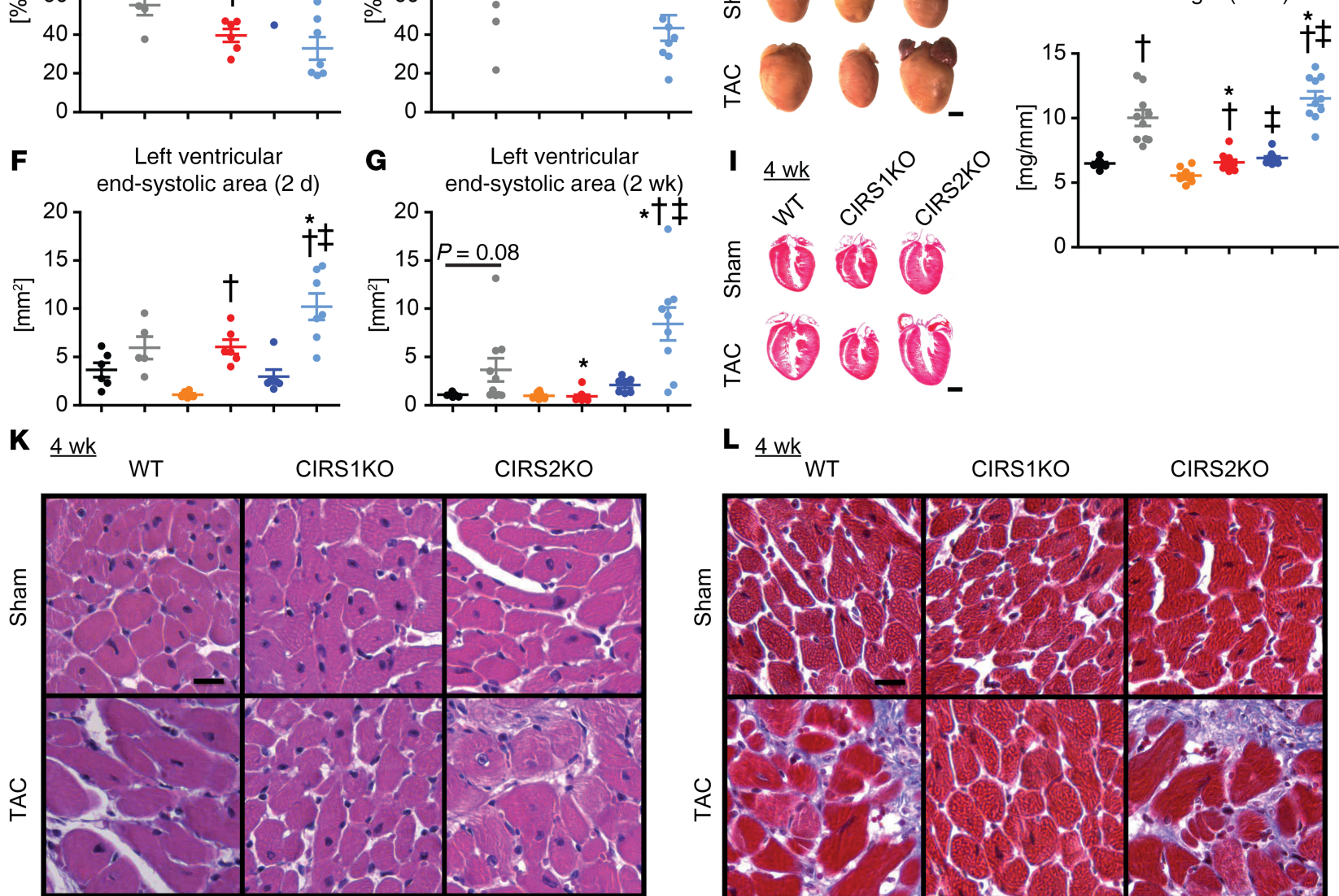

L $\underline{4 \mathrm{wk}}$
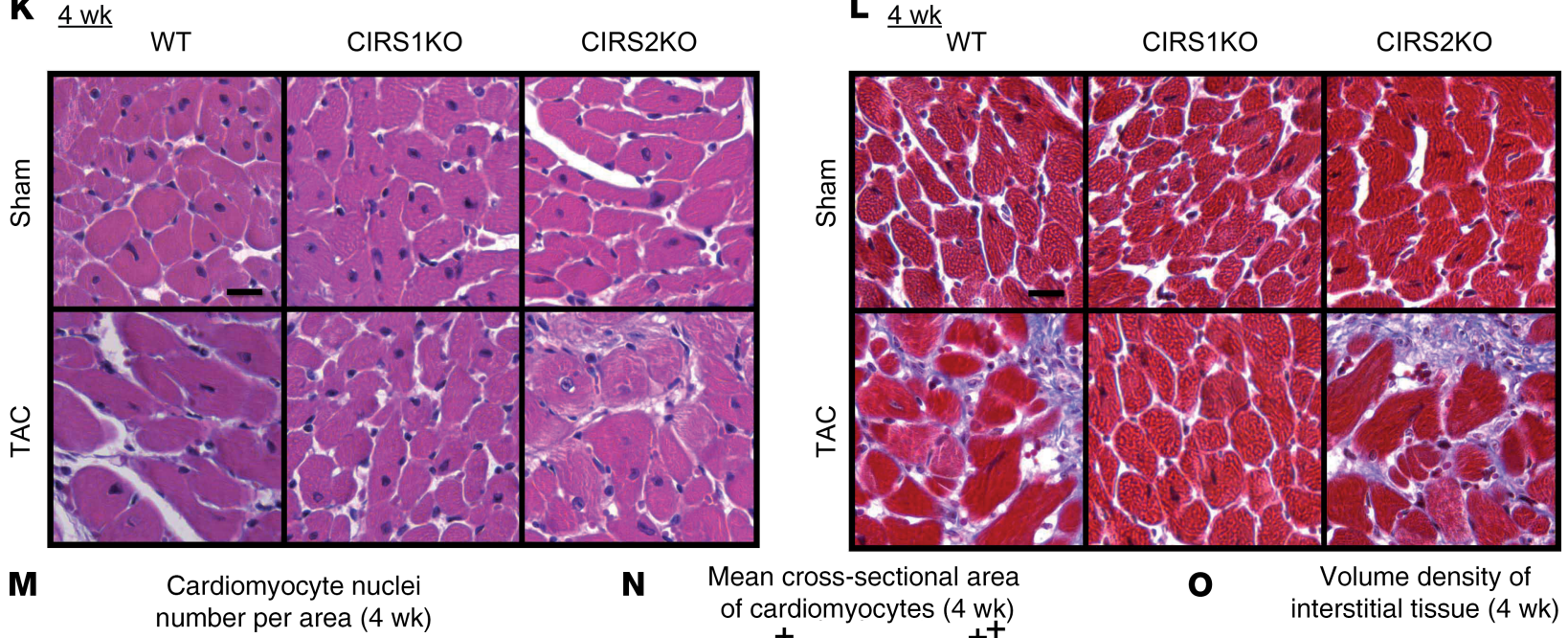

N Mean cross-sectional area
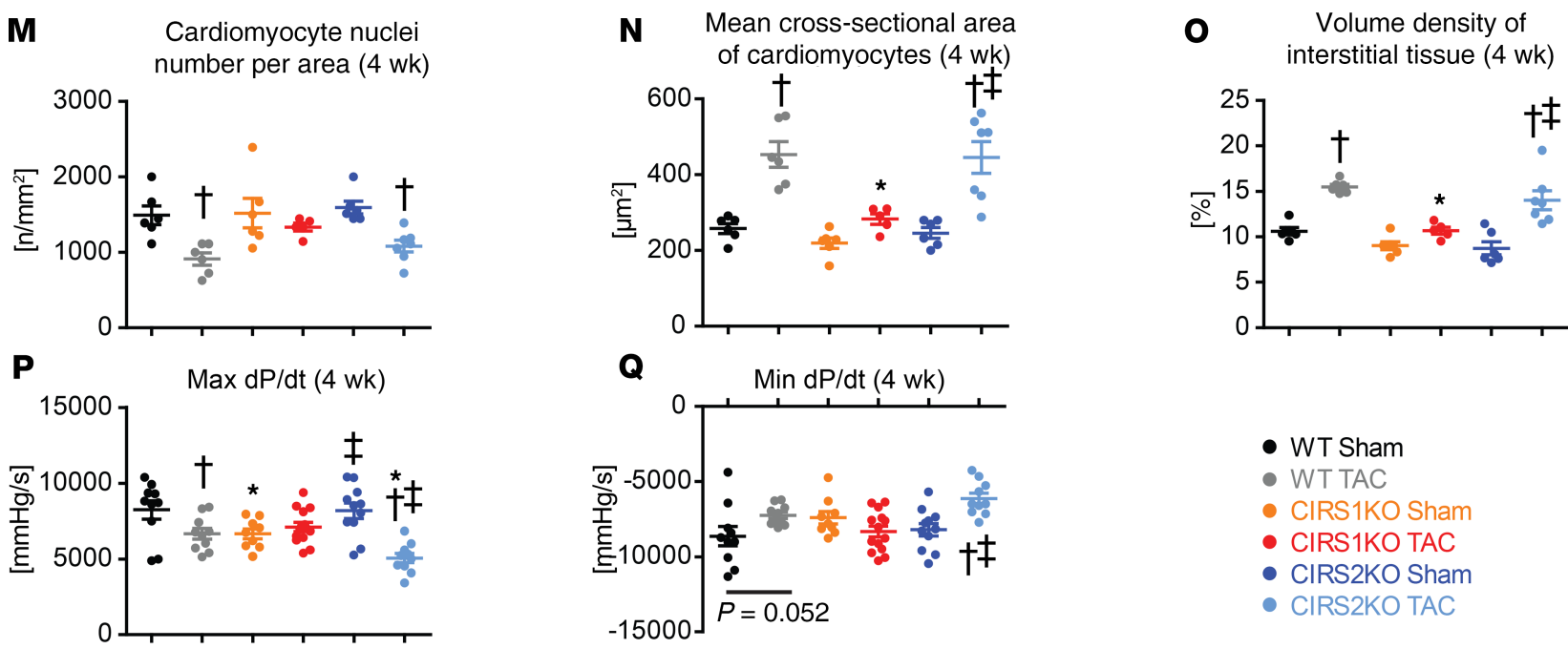

- WT Sham

- WTTAC

- CIRS1KO Sham

- CIRSIKOTAC

- CIRS2KO Sham

- CIRS2KOTAC 
Figure 1. IRS1, but not IRS2, is hyperactivated under pressure overload conditions, and IRS1 deficiency protects against heart failure in response to TAC. (A-C) Representative immunoblot and densitometry of protein levels (A and B) and tyrosine phosphorylation (C) of IRS proteins normalized to protein abundance in mouse hearts 4 weeks after TAC surgery $\left(n=7 ;{ }^{\dagger} P<0.05\right.$ vs. Sham, $t$ test). Lanes were run on the same gel but were noncontiguous. For data in panels $\mathbf{D}-\mathbf{G}, \mathbf{J}$, and $\mathbf{M}-\mathbf{Q}, 2$-way ANOVA was performed to analyze differences 4 weeks after TAC surgery by genotype, followed by Holm-Šídák post hoc analysis. Results of post hoc analyses for each comparison are summarized by symbols as defined: $\# P<0.05$ for TAC surgery, $\$ P<0.05$ for genotype, and ${ }^{\&} P<0.05$ for the interaction between TAC surgery and genotype. (D-C) Contractile function determined by ejection fraction 2 days (D) and 2 weeks (E) after Sham and TAC surgery $(n=5-12 ; \#, \$, \&$ each) and left ventricular endocardial area at end-systole 2 days (F) and 2 weeks (G) after Sham and TAC surgery ( $n=5-12 ; \#, \$, \&$ each). (H-J) Representative photographs $(\mathbf{H})$ and H\&E (I) stains of longitudinal sections of WT, CIRS1KO, and CIRS2KO hearts 4 weeks after surgery (scale bars: $2 \mathrm{~mm}$ ), and heart weights (J) normalized to tibia length $(n=10 ; \#, \$, \&)$. (K-0) Representative H\&E (K) and trichrome stains (L) (scale bars: $20 \mu \mathrm{m})$ and stereological quantification as indicated (M-0) $(n=5-7 ; \mathbf{M}, \# ; \mathbf{N}$ and $\mathbf{0}, \#, \$, \&)$. (P and $\mathbf{Q})$ In vivo hemodynamic parameters estimated by maximum rates of increase $(\mathrm{Max} ; \#, \&)(\mathbf{P})$ and decrease $(\mathrm{Min} ; \#, \&)(\mathbf{Q})$ in left ventricular pressure (dP/dt), $n=9-14$. Data shown are mean values \pm SEM. ${ }^{*} P<0.05$ vs. WT same surgery, ${ }^{\dagger} P<0.05$ vs. Sham same genotype, ${ }^{\ddagger} P<0.05$ vs. CIRS1KO same surgery.

the activation of the Akt/mTOR signaling pathway, which could, in part, mediate cardiac hypertrophy (12, 13). Activation of downstream targets of Akt — such as FoxO3, as well as mTOR and its targets - were inhibited after TAC in CIRS1KO hearts, which contrasts with the robust activation of these pathways in WT and CIRS2KO hearts (Figure 2, A-H). mTOR phosphorylation trended to be greater after TAC in CIRS2KO relative to similarly treated WT mice $(P=0.07)$. Downstream targets of mTOR such as $\mathrm{S} 6$ and 4E-BP1 phosphorylation were significantly increased in WT and CIRS2KO hearts after TAC. Interestingly, in CIRS2KO hearts, baseline phosphorylation of 4E-BP1 at Thr37/46 was increased relative to other genotypes and did not increase further following TAC. Moreover, total abundance and phosphorylation of 4E-BP1 $\gamma$ that corresponds to the phosphorylated moiety was increased in WT and CIRS2KO hearts following TAC by 1.4- to 2.6-fold $(P<0.05)$ relative to Sham but were not increased in CIRS1KO hearts (Figure 2, A-H). Isoform-specific phosphorylation of Akt was determined in response to TAC; Akt1 (1.6- to 2.3-fold, $P<0.05$ ), but not Akt2 phosphorylation, increased in WT and CIRS2KO hearts after TAC surgery, while no increase in Akt1 phosphorylation was observed in CIRS1KO hearts following TAC (Figure 2, I-M). These data indicate that IRS1 activates Akt1/mTOR signaling in response to PO.

Heterozygous deletion of Akt1 prevents activation of the mTOR signaling pathway and prevents PO-induced heart failure in IRS2-deficient hearts. We next tested the hypothesis that reduction of Akt1 signaling in the presence of preserved IRS1 signaling could attenuate LV remodeling and phenocopy the response of CIRS1KO hearts to TAC. We generated CIRS2KO mice with cardiomyocyte-specific reduction in Akt1 expression by heterozygous deletion of Akt1. These mice are annotated as CIRS2KO $\times$ Akt $1^{\text {het }}$ (IRS2 ${ }^{\text {lox/lox }} \mathrm{Akt}^{+/ / \operatorname{lox}} \alpha \mathrm{MHC}-\mathrm{Cre}^{+/-}$) and were subjected to either Sham or TAC surgery. Heterozygous deletion of Akt1 decreased the hypertrophic response in mice with intact IRS1/2 signaling or mice with IRS1 signaling alone, as observed for Akt ${ }^{\text {het }}$ and CIRS2KO $\times \mathrm{Akt}^{\text {het }}$ hearts after TAC surgery (Figure 3, A-C, and Supplemental Table 5). Similarly, transthoracic echocardiography and LV catheterization in vivo indicated reduced LV dilation and contractile dysfunction relative to WT controls and CIRS2KO hearts, which express both Akt1 alleles (Figure 3, D-G; Supplemental Table 6; and Supplemental Table 7). A pathological increase in wet lung weight (pulmonary congestion) was absent (Figure $3 \mathrm{H}$ and Supplemental Table 5), and the increase in mRNA expression for heart failure markers was diminished following Akt1 heterozygous deletion (Figure 3, I-K). Immunoblot analysis showed decreased hyperactivation of Akt/mTOR signaling pathways following heterozygous deletion of Akt1 in the face of persistent IRS1 signaling (Figure 3, L-R). Thus, heterozygous deletion of Akt1 attenuates hyperactivation of the mTOR signaling pathway and adverse LV remodeling under PO conditions independent of IRS2 expression when IRS1 signaling is preserved.

Isoform-specific hyperactivation of IRS1/Akt1 signaling in human heart samples. We examined LV tissue from failing human hearts, obtained at the time of implantation of a left ventricular assist device (LVAD). Clinical parameters are listed in Supplemental Table 8. We compared insulin signaling with LV tissue obtained from normal donor hearts not allocated for transplantation. IRS1 tyrosine phosphorylation normalized to IRS1 protein content increased by $73.7 \%(P<0.05)$ in human failing hearts vs. nonfailing donors, while IRS2 tyrosine phosphorylation, normalized to IRS2 protein levels, was unchanged (Figure 4, A-C). Phosphorylation of Akt1/2 (Ser473/4 and Thr308/9 sites) and S6 (Ser235/236) was increased in failing human hearts relative to nonfailing controls (1.5- to 2.5 -fold, $P<0.05$ ). Immunoprecipitation/immunoblot analysis showed increased Akt1 phosphorylation at Ser473 $(+84.4 \%, P<0.05)$, while Akt2 phosphorylation was unchanged (Figure 4, D-G). Together, these data reveal a specific activation of IRS1/Akt1 signaling in decompensated heart failure in both mice and humans (Figure 4, H-J). 
A
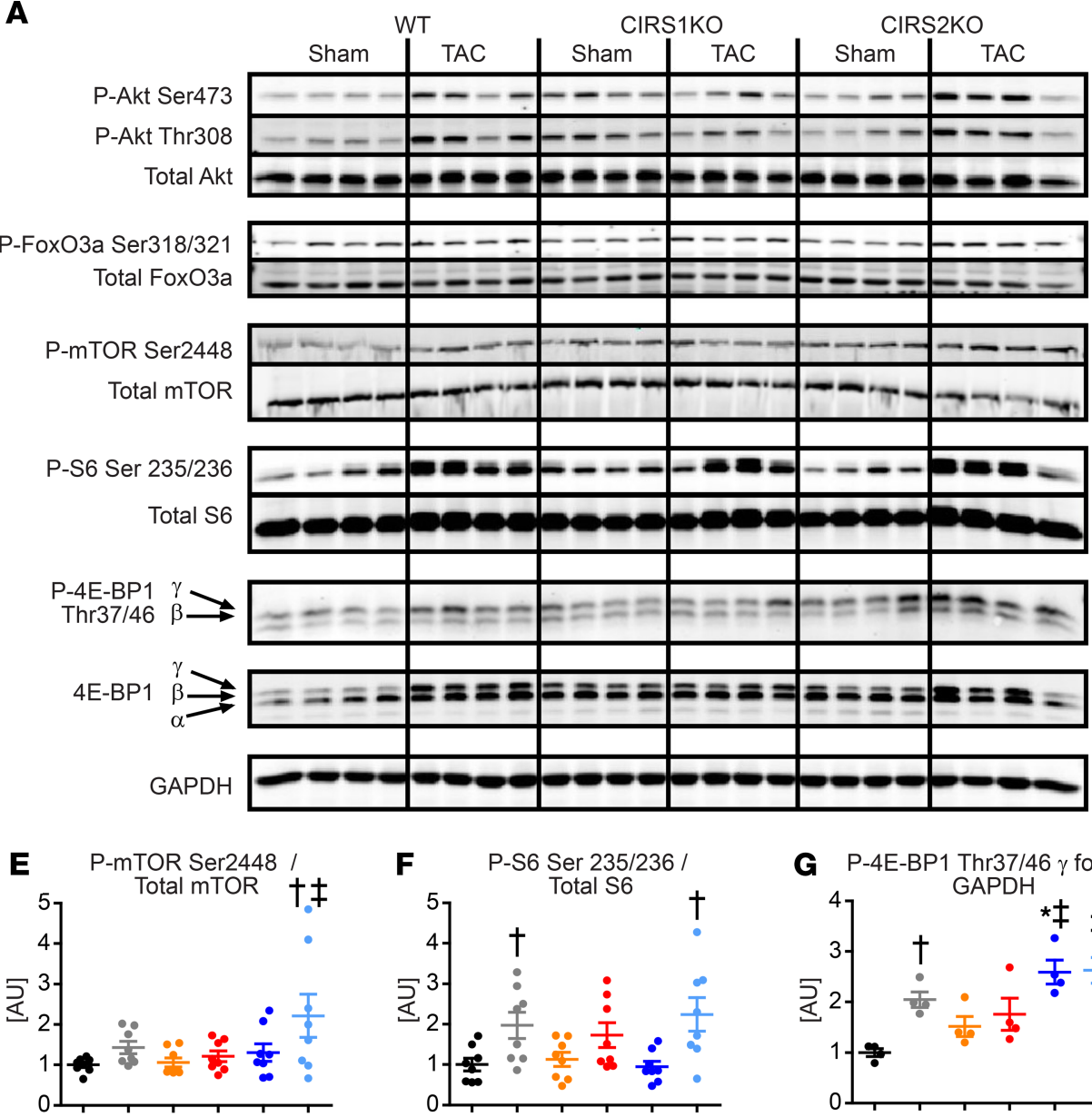

B P-Akt Ser473/ Total Akt

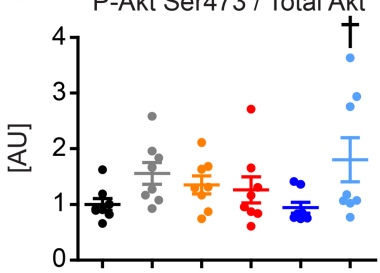

C
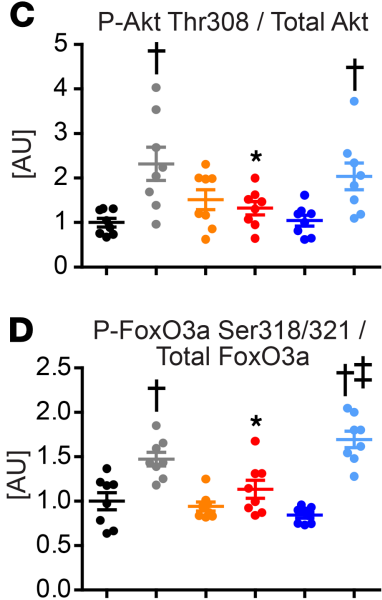

H 4E-BP1 $\gamma$ form / GAPDH

G P-4E-BP1 Thr37/46 $\gamma$ form /
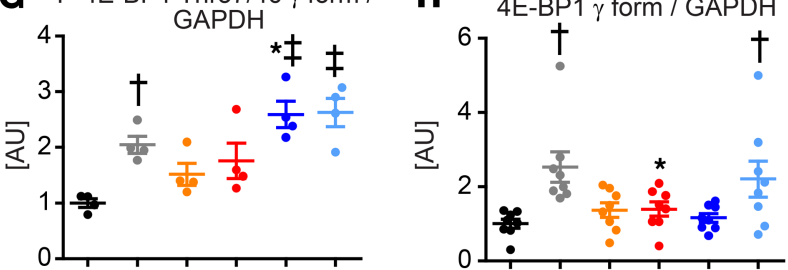

I

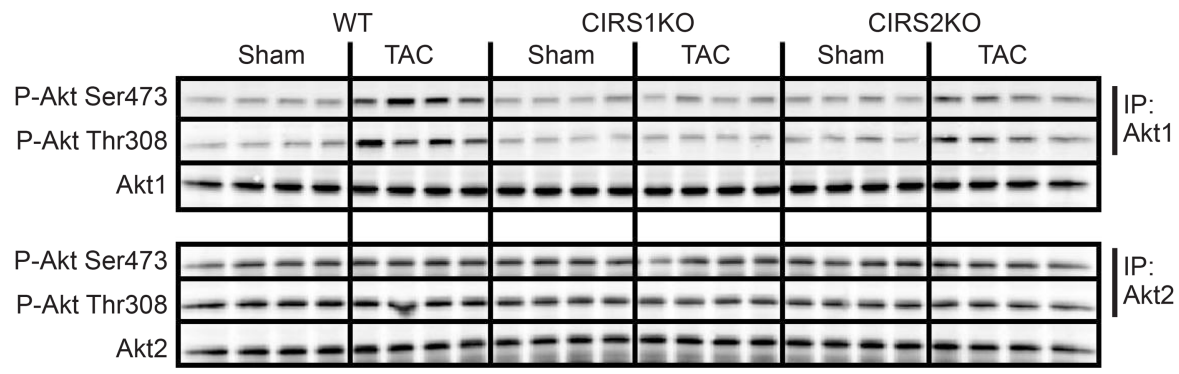

J

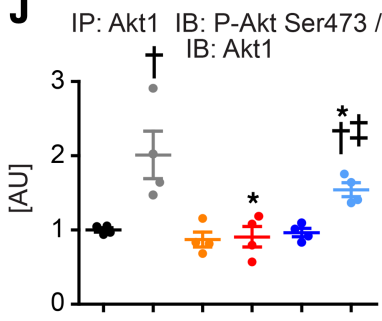

K

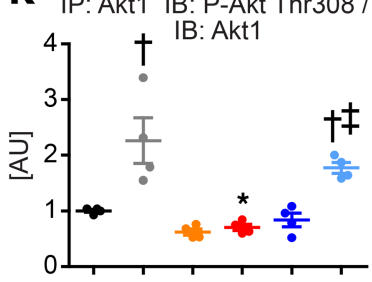

L IP: Akt2 IB: P-Akt Ser473 /

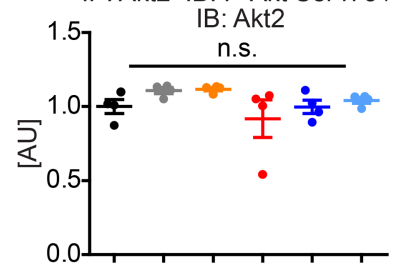

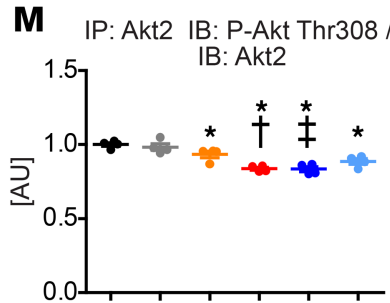

- WT Sham

- WTTAC

- CIRS1KO Sham

- CIRS1KO TAC

- CIRS2KO Sham

- cIRS2KO TAC

Figure 2. Deletion of IRS1 prevents hyperactivation of Akt1/mTOR signaling in response to pressure overload 4 weeks after TAC. Two-way ANOVA was performed to analyze differences 4 weeks after TAC surgery by genotype, followed by Holm-Šídák post hoc analysis. Results of post hoc analyses for each comparison are summarized by symbols as defined: ${ }^{\#} P<0.05$ for TAC surgery, ${ }^{\$} P<0.05$ for genotype, and ${ }^{\&} P<0.05$ for the interaction between TAC surgery and genotype. (A-H) Representative immunoblots in ventricle homogenates of WT, CIRS1KO, and CIRS2KO mice post-TAC surgery (A) and densitometric quantification of P-Akt Ser473/Total Akt (\#) (B), P-Akt Thr308/Total Akt (\#,\&) (C),P-Fox03a Ser318/321/Total Fox03 (\#,\$,\&) (D), P-mTOR Ser2448/Total mTOR (\#,\$) (E), P-S6 Ser 235/236/Total S6 (\$) (F),P-4E-BP1 Thr37/46 $\gamma$ form/CAPDH (\$,\&) (G), and 4E-BP1 $\gamma$ form/GAPDH (\#,\&) (H); $n=$ 4-8 each. (I-M) Representative immunoblots following immunoprecipitation for Akt1 or Akt2 (I) and densitometric quantification of P-Akt Ser473/Akt1 $(\#, \$, \&)(\mathrm{J})$, P-Akt Thr308/Akt1 (\#,\$,\&) following immunoprecipitation for Akt1 (K), P-Akt Ser473/Akt2 (\&) (L), and P-Akt Thr308/Akt2 (\$,\&) (M) following immunoprecipitation for Akt2 as indicated; $n=4$ each. Data shown are mean values \pm SEM. ${ }^{*} P<0.05$ vs. WT same surgery, ${ }^{\dagger} P<0.05$ vs. Sham same genotype, ${ }^{\ddagger} P<0.05$ vs. CIRS1KO same surgery. 
- WT Sham

- WTTAC

- CIRS2KO Sham

- CIRS2KO TAC

- Akt1 ${ }^{\text {het }}$ Sham

- Akt1 het TAC

- CIRS2KO $\times \mathrm{Akt}^{\text {het }}$ Sham

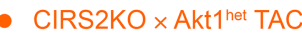

A

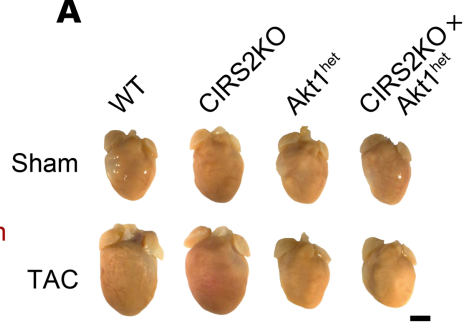

B

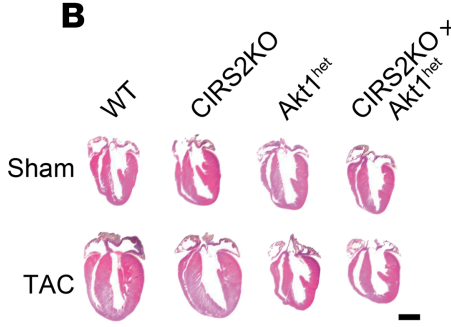

C

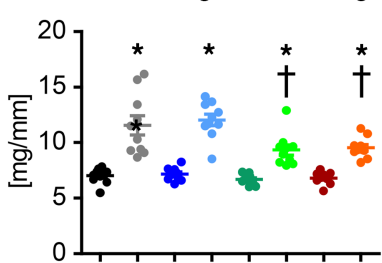

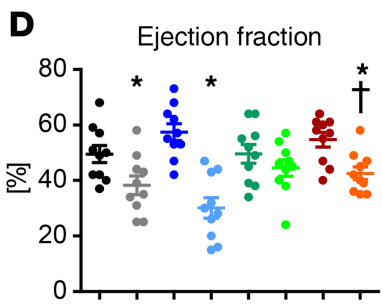

E

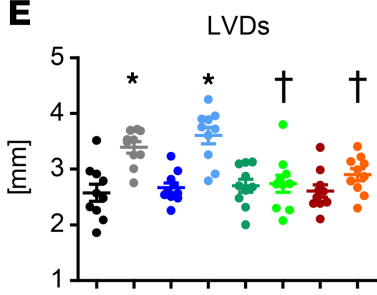

$\mathbf{F}$

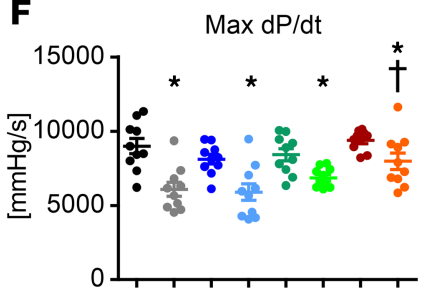

J

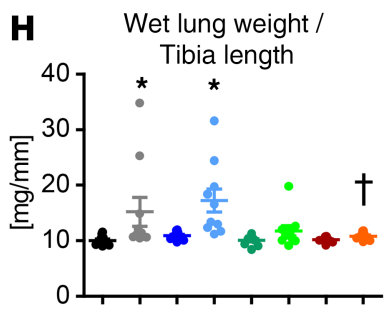

I

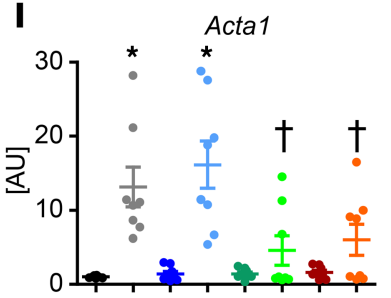

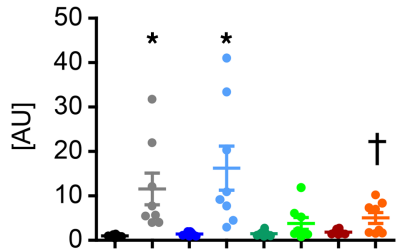

$\mathbf{L}$
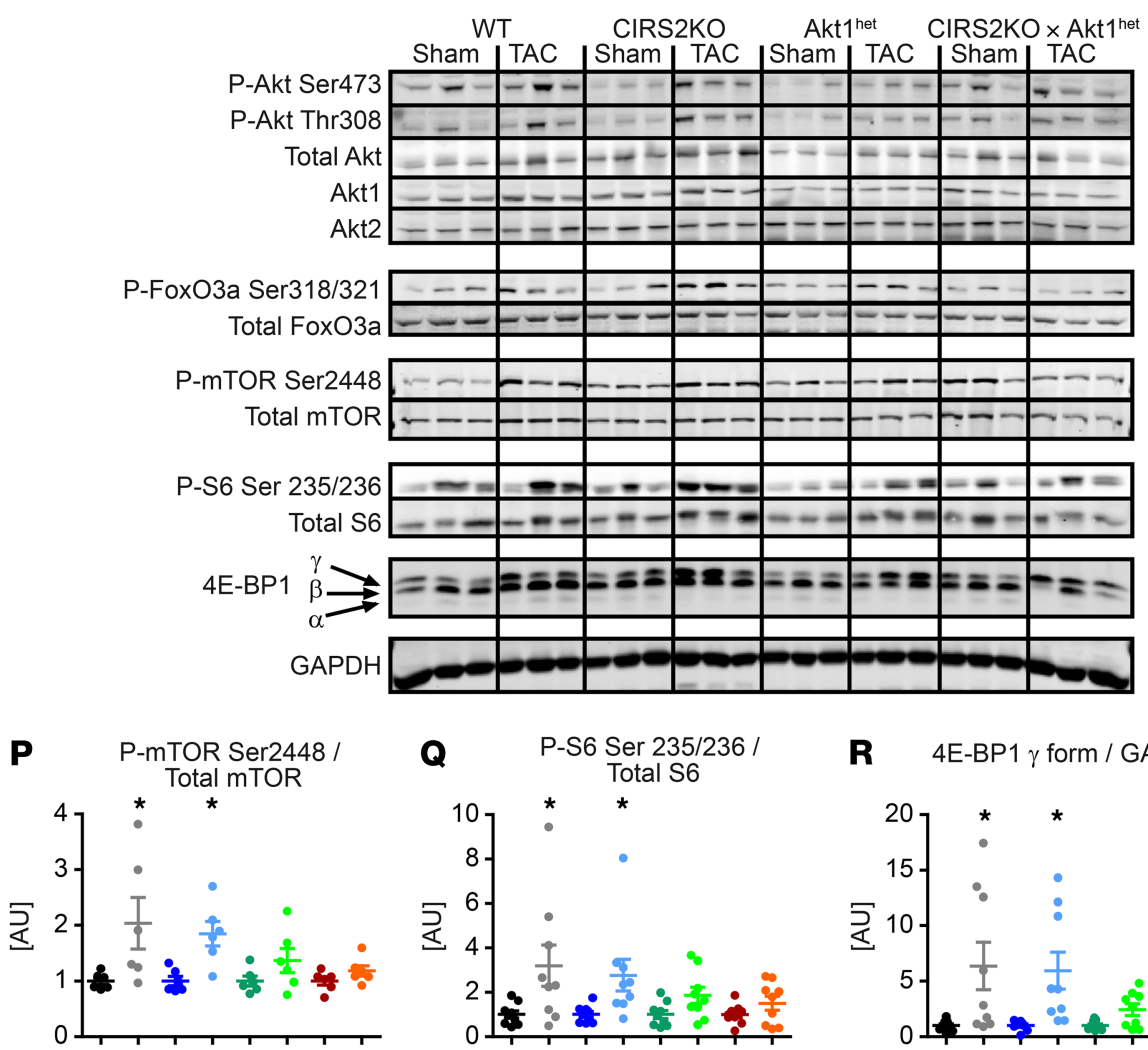

R 4E-BP1 $\gamma$ form / GAPDH

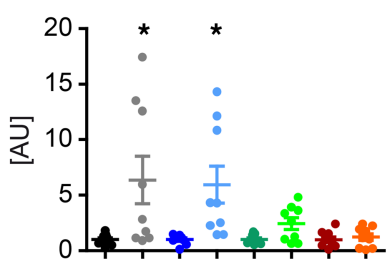

G

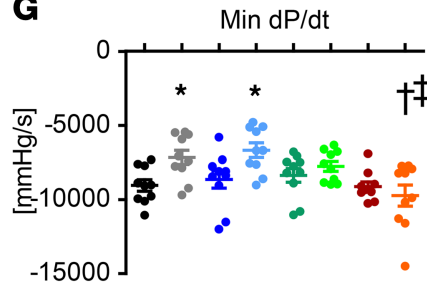

$\mathbf{K}$

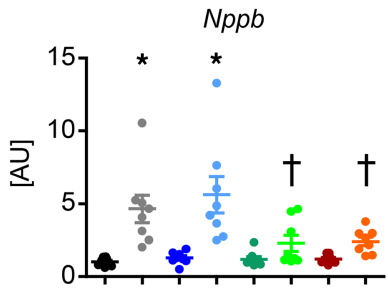

M

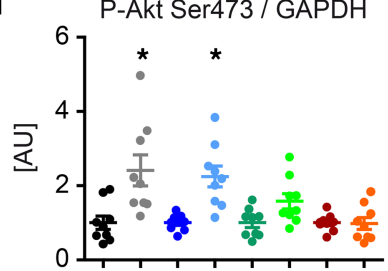

$\mathbf{N}$

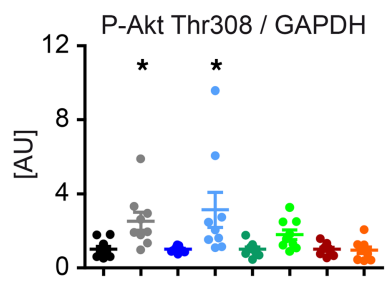

O

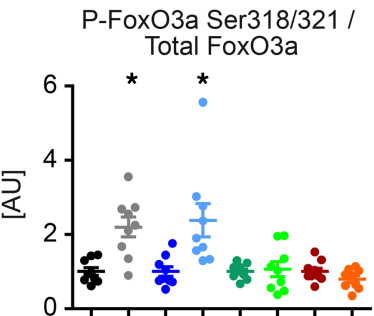

Figure 3. Heterozygous deletion of Akt1 prevents hyperactivation of the mTOR signaling pathway and protects against heart failure in IRS2-deficient hearts 4 weeks after TAC. Two-way ANOVA was performed to analyze differences 4 weeks after TAC surgery and by genotype, followed by HolmŠíák post-hoc analysis. Results of post-hoc analyses for each comparison are summarized by symbols as defined: ${ }^{\#} P<0.05$ for TAC surgery, ${ }^{\$} P<0.05$ for genotype, and \& $P<0.05$ for the interaction between TAC surgery and genotype. (A and B) Representative photographs and H\&E stains of longitudinal sections 4 weeks after surgery as indicated. Scale bars: 2 mm. (C) Heart weights normalized to tibia length ( $n=10 ; \#, \$, \&$ ). (D-K) In vivo contractile function and hemodynamic parameters as determined by ejection fraction (\#,\&) (D), left ventricular cavity diameter at systole (LVDs, \#,\$,\&) 
$(\mathbf{E})$, maximum rates of increase $(\mathrm{Max}, \#, \$)(\mathbf{F})$ and decrease $(\mathrm{Min}, \#, \$, \&)(\mathbf{C})$ in left ventricular pressure $[\mathrm{dP} / \mathrm{dt}]$, wet lung weights normalized to tibia length $(n=10 ; \#, \$)(\mathbf{H})$, and mRNA expression of Acta1, Nppa, and Nppb (I-K) (\#,\$,\& each) normalized to Cphn 4 weeks after surgery, $n=8$. (L-R) Representative immunoblots in ventricle homogenates 4 weeks after TAC surgery and densitometric quantification as indicated (presented as a fold change relative to Sham surgery same genotype; $n=6-9$ ). Data shown are mean values $\pm \mathrm{SEM} .{ }^{*} P<0.05$ vs. Sham same genotype, ${ }^{\dagger} P<0.05$ vs. Akt 1 WT same surgery, ${ }^{\ddagger} P<0.05$ vs. IRS2 WT same surgery.

Kinomic profiling identifies hyperactivation of PKG signaling in CIRS1KO hearts after TAC. To gain additional insights into IRS isoform-specific activated kinases and signaling pathways, we examined global kinase activity in WT, CIRS1KO, and CIRS2KO hearts after Sham and TAC surgery using a high-throughput peptide microarray system (kinomic profiling). Phosphorylation profiles depicted as a clustered heatmap are presented in Supplemental Table 9. An upstream kinase algorithm was utilized to generate a list of protein kinases predicted to be involved in phosphorylating the selected amino acid residues (Supplemental Table 10). The identified kinases were mapped using 2- and 3-step models to identify annotated interactions between the kinases (Figure 5, A and B; Supplemental Figure 2; and Supplemental Figure 3). In accordance with Western blot data, the algorithms identified mTOR as the top kinase activated in WT hearts after TAC surgery. Interestingly, PKG2 and PKG1 were the top 2 kinases activated in CIRS1KO hearts following TAC (Supplemental Table 10; Figure 5, A and B; Supplemental Figure 2; and Supplemental Figure 3). No upstream kinase activation was predicted for CIRS2KO hearts after TAC surgery.

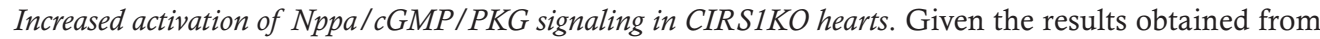
the kinomic profiling experiment, we investigated the activation status of PKG signaling in CIRS1KO hearts. PKG signaling is mediated by natriuretic peptide (NP) and nitric oxide (NO) signaling pathways, which are linked to guanylate cyclases (GC) to generate the second messenger cGMP. NP receptor-coupled particulate GC (pGC), which is located at the cellular membrane, and NO stimulated soluble GC (sGC), which is located in the cytosol, generate the downstream effector cGMP that activates PKG signaling (14). Interestingly, relative to WT and CIRS2KO mice, neither Nppa and Nppb mRNA expression were changed in CIRS1KO hearts under basal conditions, and neither were induced in response to TAC (Figure 5, C and D). Importantly, cGMP levels and PKG activity increased in CIRS1KO hearts after TAC surgery to a greater extent relative to WT and CIRS2KO hearts (Figure 5, E and F).

Phosphodiesterases (PDEs) degrade cAMP and cGMP by hydrolyzing them to their 5'-monophosphate form. A total of $11 \mathrm{PDE}$ isoforms has been identified. Of the cGMP-selective PDEs described, only PDE5 and PDE9 have been detected in cardiac tissue. PDE9a has previously been shown to degrade NP-stimulated cGMP in the heart (14). Because of the increase in cGMP levels and PKG activity observed in CIRS1KO hearts after TAC, we sought to determine if PDE9a activity was reduced in CIRS1KO mice. Adult cardiomyocytes from IRS $1^{\text {lox/lox }}$ and CIRS1KO mice were isolated and virally transduced to overexpress a FRET-based cGMP sensor. After stimulation of these cells with atrial natriuretic peptide (ANP), slightly but significantly decreased levels of cGMP were detected in cardiomyocytes obtained from CIRS1KO mice relative to IRS1 $1^{\text {lox/lox }}$ controls (Figure 5G). Following PDE9a inhibition with PF-04447943, there was a dose-dependent increase in ANP-stimulated cGMP levels in both groups. However, the magnitude of the increase was attenuated in CIRS1KO relative to IRS1 ${ }^{\text {lox } / \text { lox }}$ cardiomyocytes, consistent with reduced PDE9a activity in CIRS1KO hearts. ANP-stimulated cGMP production was increased in the presence of the PDE5 inhibitor Sildenafil and the PDE3 inhibitor Cilostamide in cardiomyocytes from CIRS1KO mice relative to controls (Supplemental Figure 4), thus indicating increased PDE5 and PDE3 activity in cardiomyocytes from CIRS1KO mouse hearts. This might be predicted to increase the catabolism of cGMP to GMP and lower cGMP levels and PKG activity in CIRS1KO hearts. This could account for the lower basal levels of cGMP in CIRS1KO hearts. However, the increase in cGMP levels and PKG activity observed in CIRS1KO hearts after TAC supports a relative predominant role for decreased PDE9a activity to account for differences in ANP-stimulated cGMP in CIRS1KO hearts (Figure 5H).

Isolated working hearts from Sham-operated WT, CIRS1KO, and CIRS2KO mice showed similar rates of glycolysis, glucose oxidation, and palmitate oxidation. Palmitate oxidation was suppressed equivalently after TAC in all genotypes (Supplemental Figure 5). This suggests that the cardioprotection observed in CIRS1KO hearts is independent of cardiac substrate utilization. To further delineate the mechanisms contributing to the recovery in contractile function in CIRS1KO hearts after TAC surgery (Figure 1, D-G), we performed differential gene expression analysis using RNA sequencing (RNA-seq) (Figure 6 and Supplemental Figure 6). Principal component analysis revealed that gene expression patterns for Sham and TAC 
A

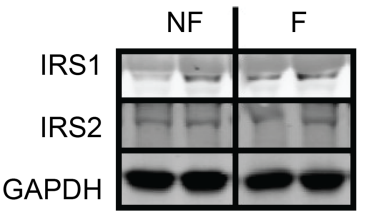

D
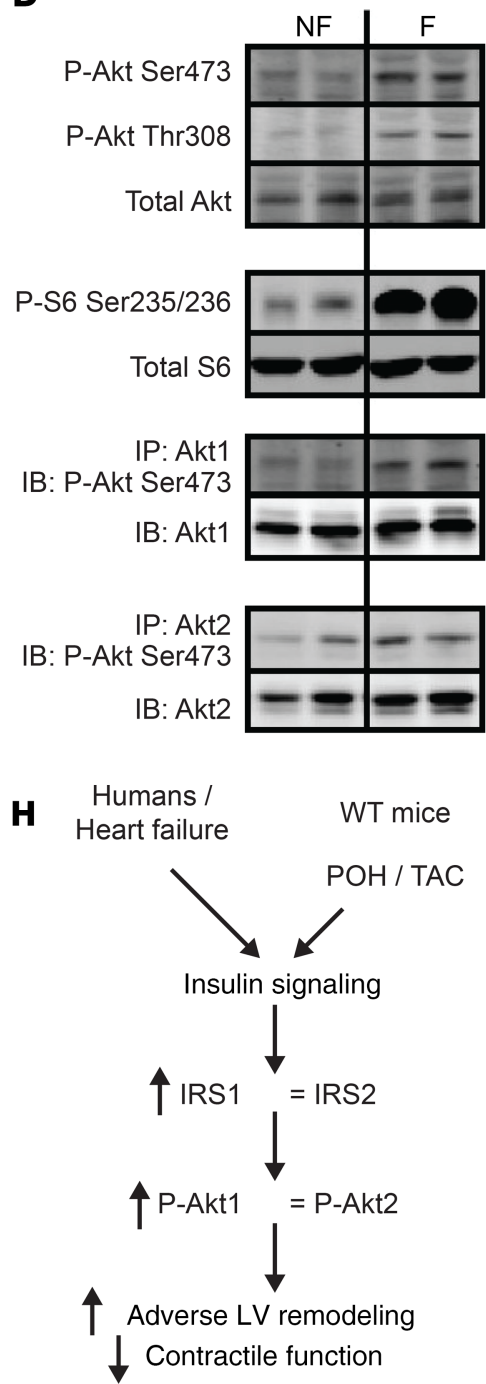

B

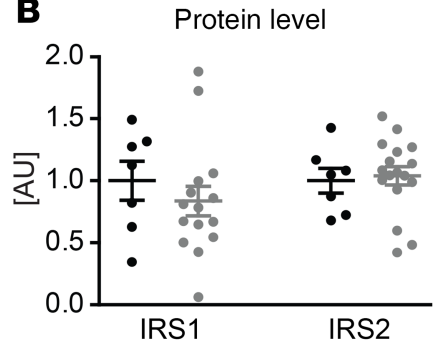

C Tyrosine phosphorylation /

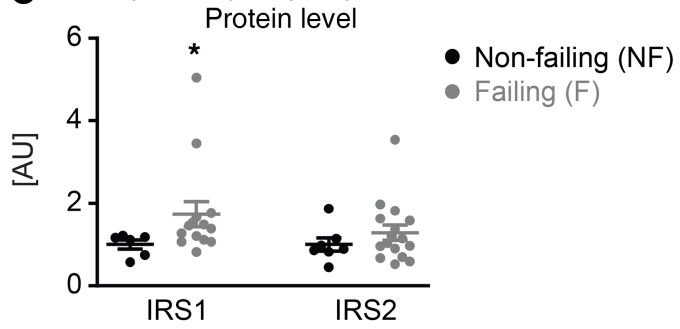

E

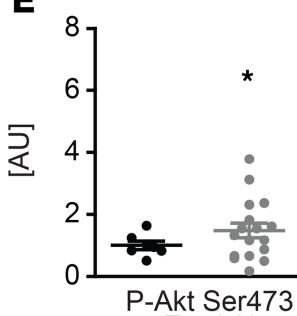

P-Akt Ser473

Total Akt

F

IP: Akt1 IB: P-Akt Ser473/

IB: Akt1

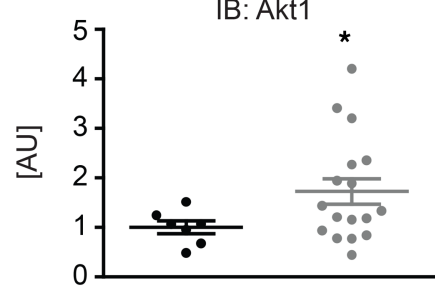

I

CIRS1KO

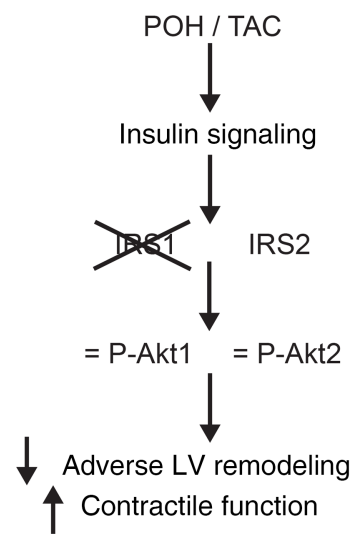

G

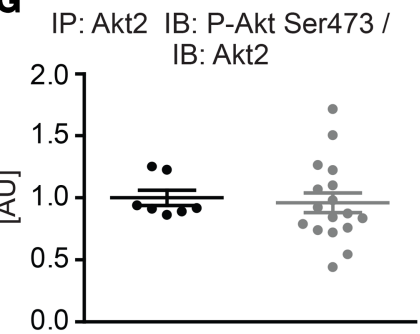

J

CIRS2KO $\times A k t 1^{\text {het }}$

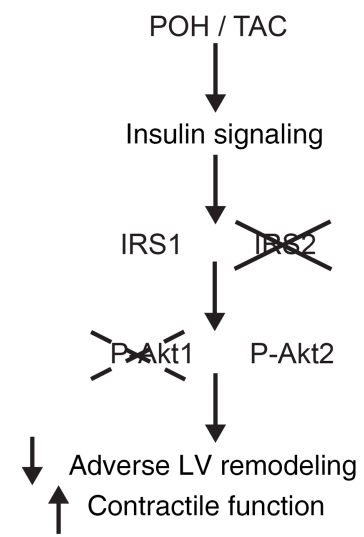

Figure 4. Isoform-specific hyperactivation of IRS1-Akt1 signaling in human heart failure. (A and B) Representative immunoblot and densitometric analysis of protein levels. (C) Tyrosine phosphorylation of IRS proteins measured by ELISA and normalized to protein abundance in human heart failure samples ( $n=14-16)$ and nonfailing controls $(n=6-7)$. ( $\mathbf{D}$ and $\mathbf{E})$ Representative immunoblots and immunoprecipitations in homogenates from human heart failure samples and nonfailing controls and densitometric quantification of immunoblots as indicated. (F and $\mathbf{G})$ P-Akt Ser473 following Akt1 immunoprecipitation normalized to total Akt1 protein and P-Akt Ser473 following Akt2 immunoprecipitation normalized to total Akt2 protein. Lanes were run on the same gel but were noncontiguous. Data shown are mean values \pm SEM presented as a fold change relative to nonfailing controls, $n=7-15$. ${ }^{*} P<0.05$ vs. nonfailing controls. (H) Schematic summary of signaling changes in human heart failure samples and murine WT hearts after TAC relative to nonfailing controls or WT Sham controls, respectively. Note that IRS1 is hyperphosphorylated in both WT mice after TAC and human heart failure samples. IRS1 total protein abundance is also increased in WT mice after TAC. (I and J) Summary of changes in CIRS1KO and CIRS2KO $\times \mathrm{Akt}^{\text {het }}$ hearts after TAC relative to TAC operated WT controls. 

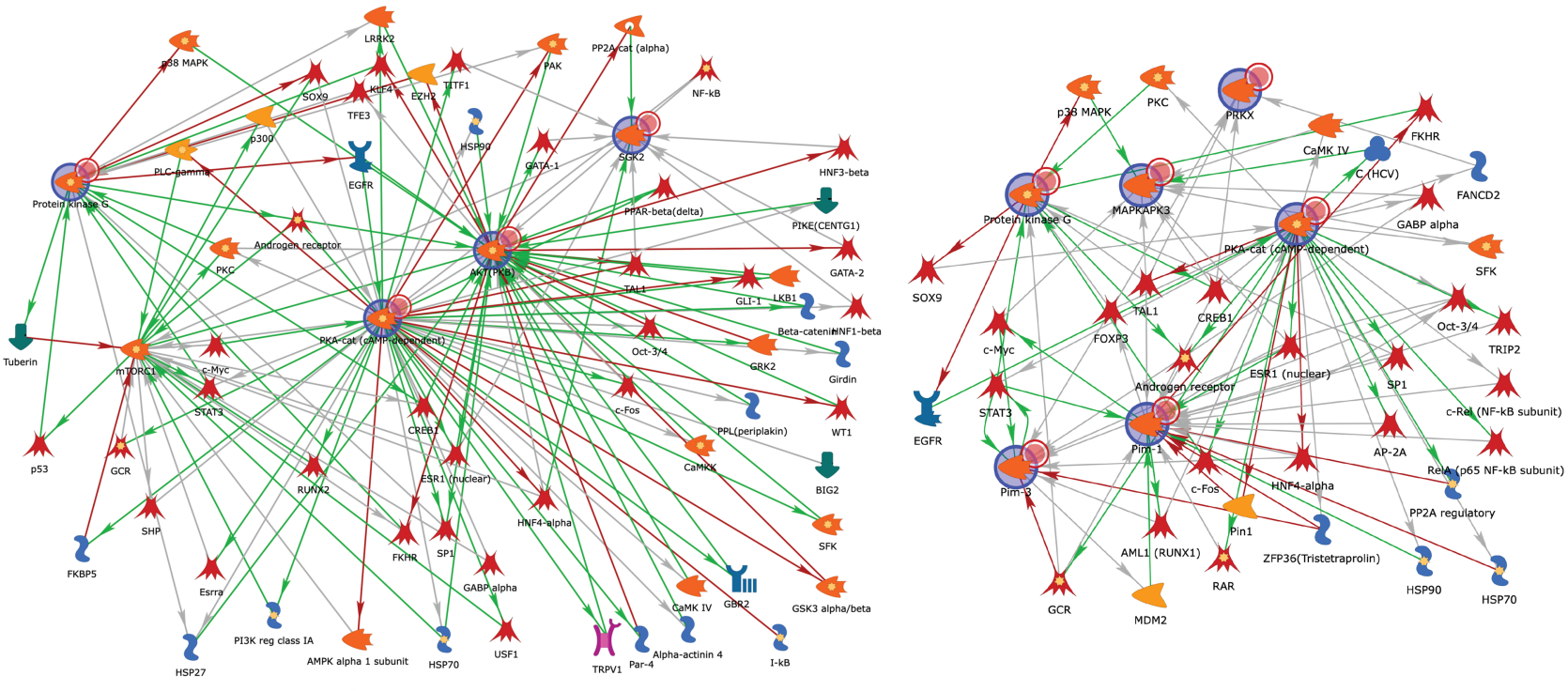

$\varangle$ Generic enzyme

\& Generic kinase

a Protein

2 Generic binding protein

$$
\begin{aligned}
& \text { Transcription factor } \\
& \text { Receptor with enzyme activity } \\
& \text { Regulator }
\end{aligned}
$$

$$
\begin{aligned}
& \longrightarrow \text { Postive effect } \\
& \longrightarrow \text { Negative effect } \\
& \longrightarrow \text { Unspecified effect }
\end{aligned}
$$

- WT Sham

- WTTAC
- CIRS1KO Sham

- CIRS1KO TAC
- CIRS2KO Sham

- cIRS2KO TAC

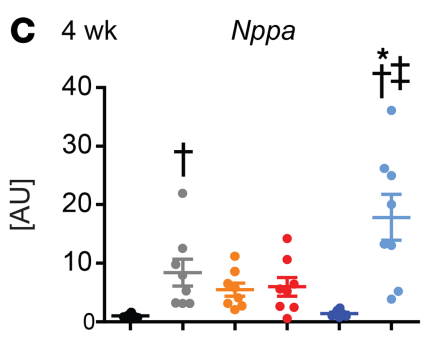

G Basal PF-04447943

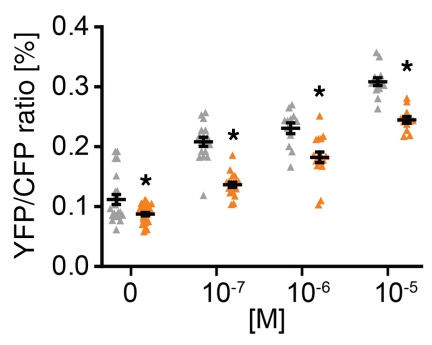

D 4 wk Nppb

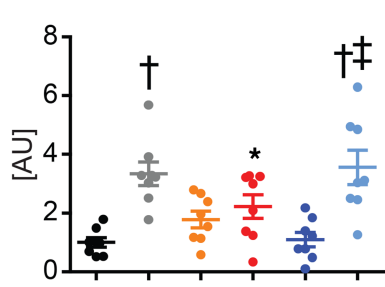

E 4 wk
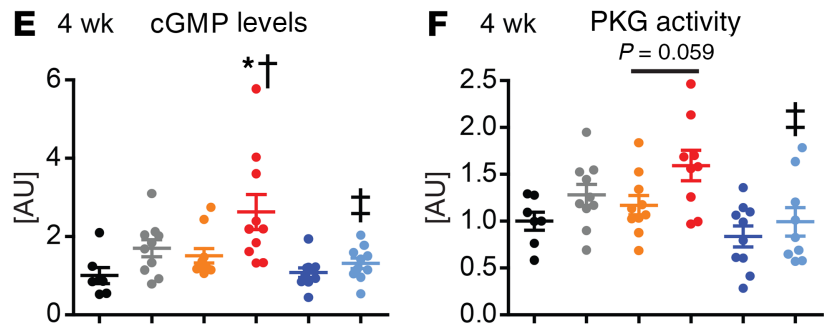

H

CIRS1KO

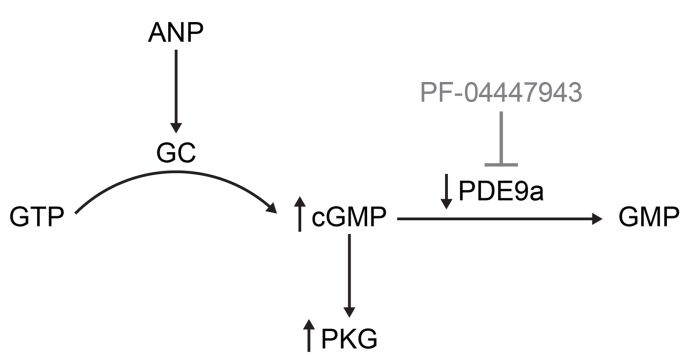

Figure 5. Increased cGMP/PKG signaling in CIRS1KO hearts. (A and B) Two-step network for TAC regulated kinase pathways in WT and CIRS1KO hearts relative to Sham controls $(n=6)$. The proteins from which the phosphosubstrates were derived were uploaded to GeneGo MetaCore (portal.genego.com) for network modeling. A Djikstras Shortest Paths algorithm allowing 1-step connections between uploaded kinases was used to generate the network for TAC altered kinases. Blue circles indicate increased nodes. No significantly regulated upstream kinase activation was identified for CIRS2KO hearts after TAC surgery. Two-way ANOVA was performed to analyze differences 4 weeks after TAC surgery by genotype, followed by Holm-Šidák post hoc analysis. Results of post-hoc analyses for each comparison are summarized by symbols as defined: ${ }^{\#} P<0.05$ for TAC surgery, ${ }^{\$} P<0.05$ for genotype, and \& $P<0.05$ for the interaction between TAC surgery and genotype. (C and $\mathbf{D})$ mRNA expression of $N p p a(\#, \$, \&)$ and $N p p b(\#, \&)$ normalized to Rps16 4 weeks after surgery, $n=8$. (E and $\mathbf{F})$ cGMP levels $(n=$ $7-10$; \#,\$) and PKG activity $\left(n=7-10\right.$; \#,\$) presented as a fold change relative to WT Sham. ${ }^{*} P<0.05$ vs. WT same surgery, ${ }^{\dagger} P<0.05$ vs. Sham same genotype, ${ }^{\ddagger} P<0.05$ vs. CIRS1KO same surgery. (C) ANP stimulated cGMP production in cardiomyocytes obtained from IRS10x/lox and CIRS1KO mice measured by using the FRET-based cGMP sensor cGi-500 with or without varying concentrations of the PDE9a-specific inhibitor PF-04447943. $n=3-5$ mice, $n=12-31$ cells/group total analyzed. Two-way ANOVA analysis was performed $(P<0.05$ for genotype, treatment, and interaction between genotype and treatment) followed by HolmŠídák post hoc analysis; ${ }^{*} P<0.05$ vs. IRS1 ${ }^{10 x / l o x}$ same PF- 04447943 concentration. (H) Proposed model for increased NP/cGMP/PKC signaling in CIRS1KO hearts. Changes in CIRS1KO hearts are presented relative to WT controls. 
A
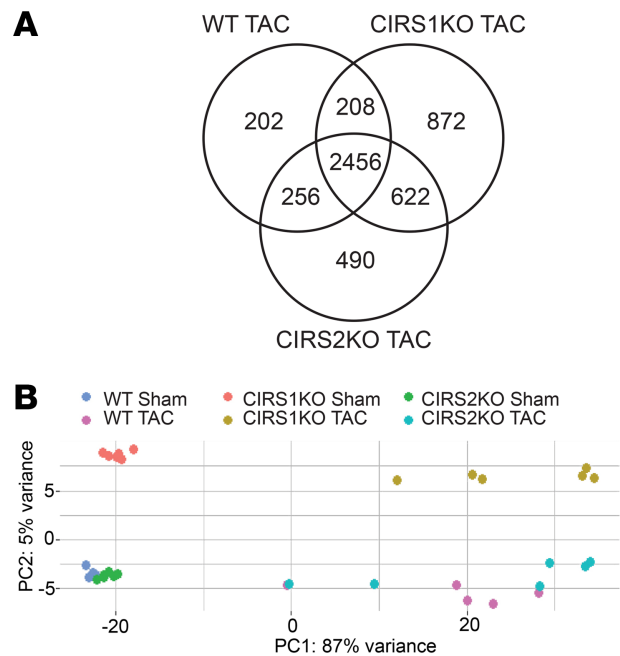

C

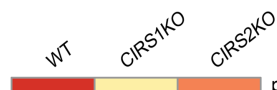

poly rl:rC-RNA

TLR3

IFNA2

STAT1

tretinoin

cytokine

cytokine
Ifnar

EIF2AK2

JAK2

CD40LG

SMARCA4

IFNL1

C3

TLR9

IFN Beta

BAX

PRKCB

Ifn

JUN

IFNA1/IFNA13

Pam3-Cys-Ser-Lys4

MYB
PAF1

N-methyl-D-aspartate

$\mathrm{N}-$ methy
DPP-23

DPP-23

GH1
PDGFB
IL27

PIN1

IRF3

enterotoxin $\mathrm{B}$

quinolinic acid

CD28

RD28

tert-butyl hydroperoxide

bromodeoxyuridine

IFN type 1

BAK1

IRF7
IL21

FGF7

IKBKG

parathyroid hormone-related protein

glycine

PDGF (family)

6-aminopyrazolopyrimidine derivative compound II

GMNN

ganglioside GM3

S-adenosylmethionine

NR4A2

ACKR2

SOCS1
TRIM24
D

WT TAC Unique

Glycoaminoglycan-protein Linkage Region Biosynthesis Agranulocyte Adhesion and Diapedesis Pyrimidine Ribonucleotides De Novo Biosynthesis Pyrimidine Ribonucleotides De Novo Blosynthesis

Pyrimidine Ribonucleotides Interconversion Th1 and Th2 Activation Pathway Role of p14/p19ARF in Tumor Suppression CXCR4 Signaling Ascorbate Recycling (Cytosolic) Notch Signaling Epithelial Adherens Junction Signaling GM-CSF Signaling CD40 Signaling TREM1 Signaling

Pattern Recognition Receptor Recognition of Bacteria and Viruses Leukocyte Extravasation Signaling

Tumoricidal Function of Hepatic Natural Killer Cells
Atherosclerosis Signaling

Tumoricidal Function of Hepatic Natural Killer Cells
Atherosclerosis Signaling

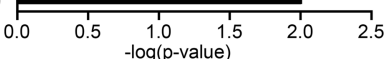

E

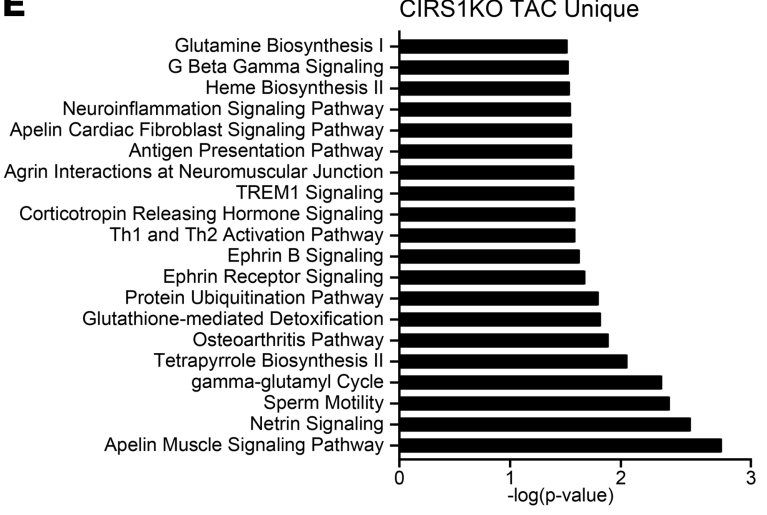

F

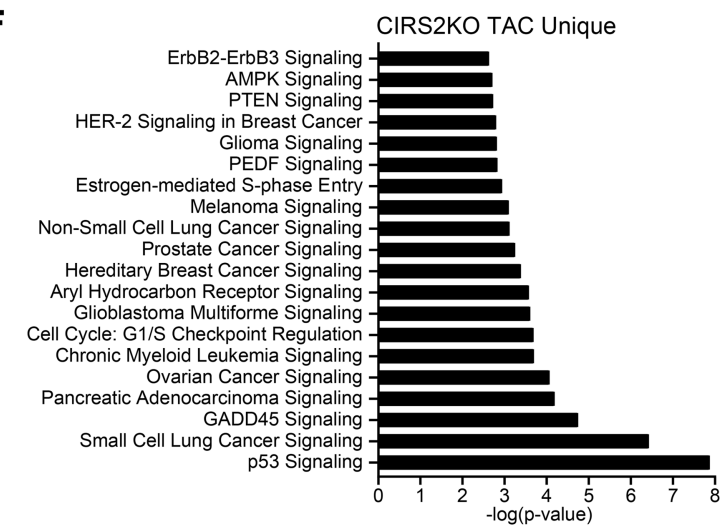

Figure 6. Gene expression 3 days after TAC as determined by RNA sequencing. (A) Venn diagram displaying the numbers of common and unique genes significantly regulated (1.5-fold change; Benjamini-Hochberg adjusted $P \leq 0.05$ ). (B) Principal component analysis to visualize global gene expression clusters by genotype and treatment. (C) Heatmap of Z-scores from ingenuity pathway analysis of genes regulated by TAC in WT, CIRS1KO, or CIRS2KO predicting the inhibition (Z-score $\leq-2$ ) or activation (Z-score $\geq 2$ ) of molecular regulators. (D-F) Ingenuity pathway analysis of genes uniquely regulated in WT, CIRS1KO, and CIRS2KO hearts 3 days after TAC. 
A
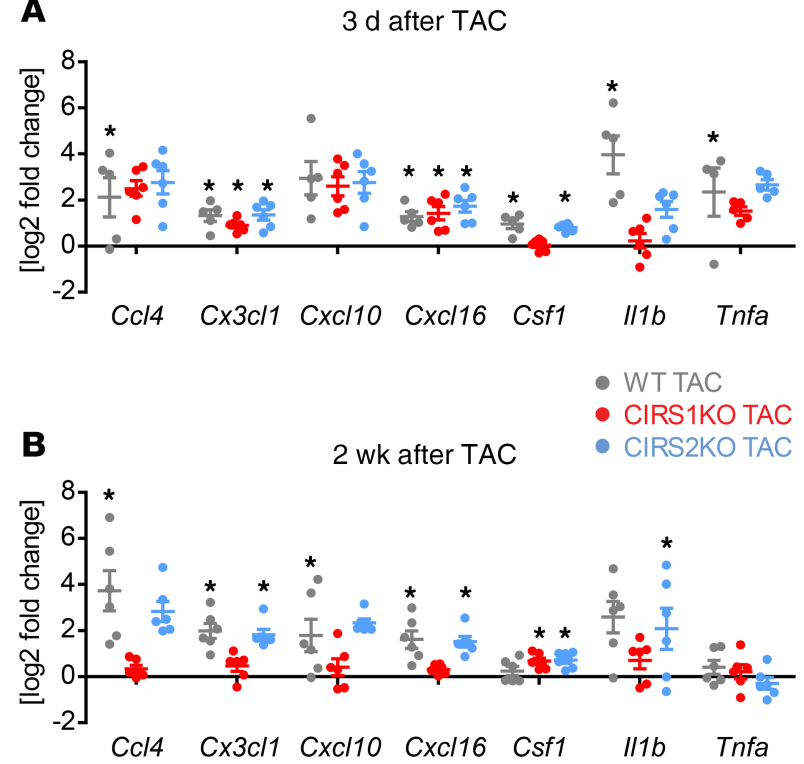

Figure 7. Attenuation of inflammatory regulators in CIRS1KO hearts after TAC. (A and B) Cytokine expression 3 days after TAC as determined by RNA sequencing $\left({ }^{*} P<0.05\right.$, Benjamini-Hochberg adjusted; TAC vs. Sham, normalized expression; $n=4-6$ ) and 2 weeks after TAC as determined by quantitative PCR ( ${ }^{*} P<0.05$, Holm-Šídák test adjusted for multiple-comparisons; TAC vs. Sham, normalized expression; $n=6$ ).

CIRS1KO samples existed in a distinct cluster from WT and CIRS2KO samples, which were overlapping (Figure 6, A and B). Ingenuity Pathway Analysis (IPA; QIAGEN Inc.) indicates that many of the genes regulated after TAC have a significant overlap with pathways known to be affected by TAC, such as oxidative phosphorylation, mitochondrial dysfunction, and fatty acid oxidation (Figure 6, C-F, and Supplemental Figure 7). This suggests that these pathways might not be modulated by IRS1 or IRS2 signaling at an early time point after TAC. IPA revealed that several mediators of inflammation or inflammatory signaling are predicted to be regulated in WT and CIRS2KO mice, but not in CIRS1KO mice. In particular, gene signatures in WT and CIRS2KO mice, consistent with activation of IFN, are predicted to be attenuated in CIRS1KO mice. Quantitative PCR (qPCR) analysis confirmed decreased expression of inflammatory markers in CIRS1KO hearts 2 weeks after TAC (Figure 7) when CIRS1KO, but not WT and CIRS2KO hearts, had recovered LV function after TAC. Interestingly, CSF1 and IL-1 $\beta$ expression was repressed in CIRS1KO mice relative to WT or CIRS2KO. This suggests a potential alteration in monocyte recruitment and/or macrophage function. Together, these data support the hypothesis that IRS1 signaling promotes proinflammatory changes in the heart following TAC.

\section{Discussion}

Hypertension, insulin resistance, and heart failure often coexist in patients with the metabolic syndrome. Furthermore, heart failure is an insulin-resistant state that is characterized by hyperinsulinemia (4). Both ischemic and nonischemic heart failure increase the risk for T2D (4). Insulin resistance is also observed in patients following myocardial infarction (15) and may predict future heart failure (16). Pathophysiologic mechanisms contributing to insulin resistance in patients afflicted with heart failure include loss of skeletal muscle mass, reduced physical activity, and hyperactivation of the sympathetic nervous system and the renin-angiotensin-aldosterone system (RAAS) (3). These changes contribute to increased circulating catecholamine levels, which stimulate adipocyte lipolysis, leading to increased plasma FFA concentrations (17). Increased concentrations of catecholamines decrease glucose utilization in skeletal muscle (18) and stimulate hepatic glycogenolysis and gluconeogenesis, resulting in increased circulating glucose and insulin levels. Importantly, proximal cardiac insulin signaling is preserved in the context of peripheral insulin resistance, as observed in high-fat diet models in rodents (19) and T2D in humans (2), while sarcolemmal GLUT4 content is decreased. Numerous studies indicate that elevated insulin levels may have adverse effects $(3,6,11)$. Several studies utilizing a variety of organisms, from yeast to mammals, have also shown that caloric restriction promotes 
longevity (20-22). A decrease in food intake may reduce circulating insulin levels and induce other beneficial processes, such as increased autophagy. Decreased insulin signaling has also been shown to extend the median lifespan and to attenuate age-dependent onset of heart failure in Drosophila melanogaster (23).

Our prior studies regarding metabolism and exercise-induced cardiac hypertrophy suggested redundant roles for IRS1 and IRS2 (10). In contrast, the present studies define distinct roles for IRS1 and IRS2 in pathological ventricular remodeling, indicating that IRS1 and IRS2 signaling in the myocardium is context dependent. Using a rodent model, we previously showed that PO resulted in hepatic insulin resistance paralleled by a compensatory increase in serum insulin concentrations. Furthermore, we showed that PO following TAC surgery in vivo or following mechanical stretch of isolated cardiomyocytes in vitro activated cardiac insulin signaling, as indicated by increased phosphorylation of IRS1/ Akt. These observations are particularly pertinent in the context of peripheral insulin resistance with hyperinsulinemia, because increased IR activation by insulin will synergize with the stretch-mediated activation of IR/IRS1 to further activate Akt/mTOR signaling pathways. Our earlier study demonstrated that reducing insulin signaling by lowering circulating insulin or by reducing IR or Akt1 expression attenuated the onset of heart failure (11). In the present study, we sought to further investigate the underlying mechanisms and to elucidate the relative contributions of IRS1 or IRS2 to this phenomenon. Our data identify isoform-specific activation of an IRS1/Akt1 signaling module both in murine hearts under PO conditions after TAC surgery and in human heart-failure samples. Importantly, insulin resistance states, such as $\mathrm{T} 2 \mathrm{D}$, increase the risk of heart failure, and heart failure contributes to insulin resistance (3). We acknowledge that approximately $50 \%$ of patients with terminal heart failure in this study were patients with diabetes. Therefore, the results obtained from human samples compared with our mouse studies have to be interpreted cautiously. While CIRS1KO hearts are protected against adverse LV remodeling and contractile dysfunction under PO conditions, CIRS2KO hearts display a phenotype similar to or more severe than WT controls. Isolated working heart experiments revealed similar substrate oxidation rates both under basal conditions and under PO conditions between the genotypes (Supplemental Figure 5). This indicates that the divergent responses observed after TAC are independent of differences in cardiac substrate metabolism. Importantly, CIRS2KO hearts maintain IRS1 signaling. In addition, we observe isoform-specific hyperactivation of Akt1 following TAC surgery, which is attenuated in CIRS1KO hearts. Our experiments also indicate that heterozygous deletion of Akt1, in the absence of IRS2 signaling, attenuates the onset of heart failure. This is supported by a previous study reporting that deletion of Akt2 had no effect under PO conditions (24), indicating that Akt2 is dispensable for the myocardial adaptations to PO. In contrast to our previous report (10), we did not observe hypertrophy in IRS2-deficient hearts under basal conditions (Figure 1J, Figure 3C, Supplemental Table 3, and Supplemental Table 5) that is supported by our stereological quantification (Figure 1N). Importantly, CIRS2KO hearts develop age-dependent cardiac hypertrophy, which was more pronounced in older CIRS2KO mice investigated in our previous report (10). We acknowledge the differences in our studies, which likely result from age-dependent hypertrophy of nonstressed IRS2-deficient hearts and the mixed genetic background of the mice used. Importantly, these differences are relatively minor compared with the significant changes in HW after TAC, which were attenuated following homozygous deletion of IRS1 and heterozygous deletion of Akt1.

Our data support the hypothesis that modulation of insulin signaling by isoform-specific reduction, but not a complete abrogation of downstream mediators of cardiac insulin signaling, is beneficial under PO conditions. This is highlighted by studies reporting accelerated heart failure in mice under PO conditions with homozygous deletion of the IR, Akt1, or Raptor $(12,25,26)$. Complete disruption of the insulin signaling cascade by combined cardiomyocyte-specific deletion of insulin/IGF1 receptors (27) or IRS1/2 (28-30) also resulted in dilated cardiomyopathy and early onset of heart failure. These studies indicate a cardioprotective role for IRS2 expression in the absence of IRS1-mediated signaling in the nonstressed heart.

In addition, we identify IRS1 signaling as a potential regulator of the NP/cGMP/PKG pathway, which identifies a potentially novel mechanism linking insulin signaling and heart failure. Our in vitro studies indicate decreased PDE9a activity in cardiomyocytes obtained from CIRS1KO hearts (Figure 5, $\mathrm{G}$ and $\mathrm{H}$ ), which may contribute to increased abundance of cGMP and increased activation of PKG in IRS1-deficient hearts. Protein abundance of PDE9A and PKG1 were not different between the groups (Supplemental Figure 8), which suggests that regulation of their activation status could be occurring via posttranslational mechanisms. A relationship between IRS1 deficiency and activation of PKG signaling 
was also supported by an unbiased targeted kinome array approach, which revealed activation of the Akt/ mTOR signaling pathway in WT mice, contrasting with activation of PKG signaling in CIRS1KO mice. We were surprised that the kinome array did not detect increased Akt/mTOR signaling in CIRS2KO mice, although immunoblotting confirmed that this was the case. This could reflect confounding effects of IRS2 deficiency on global phosphorylation patterns that are incompletely understood, at present.

NP activation leading to generation of cGMP is cardioprotective when cGMP generation is tightly coupled with activation of PKG. Our findings in CIRS1KO mice suggest that coupling of cGMP and PKG signaling is preserved or maintained following PO. The present study also suggests that selective activation of the NP/pGC signaling in CIRS1KO hearts may represent the mechanism for increased PKG signaling, as opposed to an increase in NO/sGC-mediated signaling. NO/sGC-mediated signaling has been described to result in PKG-mediated phosphorylation of Troponin I, which desensitizes myofilaments to calcium (31-33). In the present study, phosphorylation of Troponin I at Ser23/24 was not different between the groups. Similarly, stimulatory phosphorylation of eNOS at Ser1177 (34), which increases eNOS enzymatic activity, NO production, and activation of the NO/sGC pathway, was unchanged (Supplemental Figure 9). Moreover, prior studies suggest a predominant role for PDE9 for inhibiting NP/pGC-regulated cGMP signaling (14), whereas PDE5 may preferentially degrade cGMP generated by the NO/sGC pathway (35). Genetic and pharmacological inhibition of PDE9 has been shown to be cardioprotective following PO, an effect that remained when NO signaling was blocked with L-NAME. These observations suggest that PDE9 preferentially inhibits cGMP generated by the NP/pGC pathway. Inhibition of PDE5 has also been identified to be cardioprotective in rodent models (36). While differential locations for PDE isoforms have been suggested (i.e., PDE5 at myofilaments and PDE9 near transverse tubules), analyses of transcription factor activation and phosphoproteome expression patterns revealed partially overlapping downstream targets for PDE5 and PDE9 (37).

Our in vitro studies indicate increased PDE5 and PDE3 activity in cardiomyocytes from CIRS1KO mouse hearts, as evidenced by increased ANP-stimulated cGMP production in the presence of the PDE5 inhibitor Sildenafil and the PDE3 inhibitor Cilostamide relative to controls (Supplemental Figure 4). This could account for the lower basal levels of cGMP in CIRS1KO hearts. However, the increase in cGMP levels and PKG activity in CIRS1KO hearts suggests a relative predominant role for decreased PDE9a activity in the cGMP response to increased NP signaling. The relative roles of PDE isoforms, along with the underlying mechanisms leading to decreased PDE9a activity in CIRS1KO and the mechanisms linking IRS1 signaling with the activity of PDE9a in the heart, require further investigation.

Numerous studies identify the NP/cGMP/PKG signaling pathway as an attractive candidate for the pharmacological treatment of heart failure. A number of therapeutic compounds targeting this pathway are already commonly used to treat other diseases, such as pulmonary hypertension. This pathway also represents the target of an emerging new class of drugs for the treatment of chronic heart failure - namely, neprilysin inhibitors, such as Sacubitril, which prevent the degradation of NPs and enhance pGC/cGMP signaling. Importantly, decreased circulating NP levels are observed in obese patients (38). NP deficiency has been implicated in the development of insulin resistance and T2D (39), which both increase the risk of heart failure. Thus, our data provide a potential mechanistic link between myocardial insulin signaling and this critical cardioprotective pathway.

The data obtained from the RNA-seq experiment reveal attenuation of inflammatory signaling pathways following TAC in CIRS1KO mice. In particular, IFN signaling is predicted to be repressed. This is significant because IRS proteins can serve as signaling intermediates downstream of IFN (40-42). IFNs are cytokines that have diverse effects on the immune system. IFN- $\gamma$ is of interest because it is important in the function of Th1. These T cells have been implicated in the transition from hypertrophy to heart failure after TAC (43). Furthermore, activated T cells and specifically Th1 are increased in the blood and LV of heart-failure patients (44-46). Mice deficient in T cells, or mice in which costimulation has been blocked, display preserved cardiac function, decreased hypertrophy, and attenuated fibrosis (46, 47). Thus, blockade of $\mathrm{T}$ cell activation could be therapeutically beneficial in heart-failure patients. Our data suggest that cardiomyocyte IRS1 may be a critical mediator of T cell-mediated mechanisms that contribute to cardiac remodeling by serving as a signaling intermediate of IFN action.

In summary, the present study identifies the IRS1/Akt1 axis, independent of IRS2 and Akt2 signaling, as a key regulator of adverse LV remodeling that exacerbates contractile dysfunction and heart failure. Additional cardioprotective effects identified in CIRS1KO hearts include induction of NP/cGMP/PKG 
signaling and attenuated proinflammatory signaling, which may promote myocardial recovery following induction of PO. Together, these data identify a critical role for IRS1-mediated signaling pathways in exacerbating heart failure in both mice and humans, and they provide a molecular mechanism linking insulin signaling to heart failure.

\section{Methods}

Animals. CIRS1KO and CIRS2KO mice were generated using Cre/loxP recombination as previously described, and IRS2 $2^{10 x / l o x}$ mice were used as WT controls for the single KO experiments, unless otherwise indicated (10). For the CIRS2KO $\times \mathrm{Akt}^{\text {het }}$ cross experiments, $\mathrm{Akt}^{\text {het }}$ mice $\left(\mathrm{Akt}^{+/ \mathrm{lox}}: \alpha \mathrm{MHC}-\mathrm{Cre}^{+/-}\right)$were generated by cross-breeding mice harboring homozygous Akt1 alleles flanked by loxP-sites (Akt $1^{\text {lox/lox }}$ ) with mice expressing $\alpha \mathrm{MHC}-\mathrm{Cre}^{+/-}$. CIRS2KO $\times \mathrm{Akt}^{\text {het }}$ mice (IRS2 $2^{\text {lox } / \text { lox }} \mathrm{Akt}^{+/ \text {lox }} \alpha \mathrm{MHC}-\mathrm{Cre}^{+/-}$) were generated by cross-breeding CIRS2KO mice (IRS2 ${ }^{\text {lox/lox }} \alpha \mathrm{MHC}-\mathrm{Cre}^{+/-}$) with mice harboring homozygous Akt1 alleles flanked by loxP-sites (Akt $1^{\text {lox/lox }}$ ). CIRS2KO and CIRS2KO $\times \mathrm{Akt}^{\text {het }}$ mice originated from this mating in subsequent generations. WT controls did not express $\alpha \mathrm{MHC}-\mathrm{Cre}$. The generation of transgenic mice for IRS1 $1^{\text {lox } / \text { lox }}$ IRS2 $2^{\text {lox/lox }}, \mathrm{Akt}^{\text {lox } / \mathrm{lox}}$, and $\alpha \mathrm{MHC}-\mathrm{Cre}^{+/-}$has been previously reported, and genotyping was performed as described before $(10,48)$. Mice were maintained on a C57BL/6/129Sv mixed genetic background, and littermate controls were used for all comparisons. Studies were performed in male animals. Animals were housed with a 12-hour light/12-hour dark cycle at $22^{\circ} \mathrm{C}$ with free access to food and water.

Human samples. Human heart samples used in this study were obtained from patients with end-stage heart failure, treated at the University of Utah, and were procured at the time of LVAD implantation. Samples were obtained from LV apex. Control hearts not allocated for transplantation due to noncardiac reasons were used as controls.

TAC. TAC was performed at the age of 6 weeks or 8 weeks, as described in detail in the Supplemental Methods and all mice achieved a similar increase in the pressure gradient, which was independent of the genotype (Supplemental Figure 1).

Transthoracic echocardiography and hemodynamic studies. Details are provided in the Supplemental Methods.

Histology and stereological quantification. Myocardial fragments were stained with H\&E or Masson's trichrome. Stereological analysis was performed as described in detail in the Supplemental Methods.

IRS pantyrosine phosphorylation ELISA assay. Pantyrosine phosphorylation of IRS1 and IRS 2 was determined using PathScan Sandwich ELISA kits (Cell Signaling Technology) according to the manufacturer's instruction.

Immunoprecipitation and immunoblotting. Details are provided in the Supplemental Methods.

Kinomic profiling. Tissue preparation and kinomic analysis was performed as described in detail in the Supplemental Methods.

Measurement of cGMP levels and PKG activity. cGMP levels in whole heart tissue were determined using a cGMP enzyme immunoassay kit (Cayman Chemical) according to the manufacturer's instruction and normalized to tissue weight. PKG activity in whole heart tissue was determined by colorimetric analysis using a PKG assay kit (CycLex) according to the manufacturer's instruction.

Determination of cGMP PDE activity by FRET measurements. Adult cardiomyocytes from IRS $1^{\text {lox/lox }}$ and CIRS1KO mice were transduced with adenovirus carrying a transgene coding for the fluorescence resonance energy transfer-based (FRET-based) cGMP sensor cGi-500 (49). Thirty-six hours after transduction, cells were stimulated with $1 \times 10^{-5} \mu \mathrm{M}$ ANP plus or minus the PDE3 inhibitor Cilostamide, PDE5 inhibitor Sildenafil, or PDE9a inhibitor PF-04447943 at various concentrations $\left(1 \times 10^{-7} \mathrm{M}, 1 \times 10^{-6} \mathrm{M}\right.$, and $1 \times 10^{-5} \mathrm{M}$, respectively). Changes in FRET were measured using live cell microscopy as previously described $(5,50)$.

Isolated working hearts. Cardiac substrate metabolism, oxygen consumption, cardiac efficiency, cardiac power, LV developed pressure, cardiac output, and heart rate were measured in isolated working hearts obtained 4 weeks after surgery (clip method) as previously described (10). Hearts were perfused in the isolated working heart mode with Krebs Henseleit Buffer with $5 \mathrm{mmol} / \mathrm{L}$ glucose and 0.4 $\mathrm{mmol} / \mathrm{L}$ palmitate without insulin.

RNA-seq analysis. RNA-seq was performed in $n=5-6$ samples/group as described in detail in the Supplemental Methods. RNA-seq data have been deposited to the GEO database under the accession number GSE132996.

Statistics. Data are expressed as mean \pm SEM. Unpaired 2-tailed Student's $t$ tests were used to analyze data sets with 2 groups. Data sets were analyzed by 2-way ANOVA for multigroup comparisons with 
Holm-Šídák's post hoc analysis to determine significance levels between surgery and genotypes. Statistical analyses were performed using GraphPad Prism Software. For all analyses, $P<0.05$ was considered significantly different.

Study approval. All experiments involving animals were performed in accordance with protocols approved by the IACUC of the University of Utah (protocol no. 12-07009), the Carver College of Medicine of the University of Iowa (protocol nos. 1305094 and 6031709), and the University of California at Davis (protocol no. 20957). All studies involving human samples were approved by the IRB of the University of Utah and Intermountain Medical Center (Salt Lake City, Utah, USA; protocol no. 30622). All patients or their family provided written consent.

\section{Author contributions}

CR designed and performed experiments, interpreted data, and drafted and edited the manuscript. ETW designed and performed experiments, interpreted data, and edited the manuscript. ARW designed and performed experiments, interpreted data, and edited the manuscript. QS and GRR designed and performed experiments and interpreted data. BPJ, AWS, NSM, MR, WJK, KO, and KMP performed experiments and interpreted data. JCA and RMW designed and performed experiments, interpreted data, and edited the manuscript. MFW provided animal models, interpreted data, and edited the manuscript. NAD and SGD designed the clinical study, provided patient samples, and interpreted data. YKX designed experiments, interpreted data, and edited the manuscript. EDA conceptualized and supervised the project, analyzed and interpreted data, and drafted and edited the manuscript. CR and ETW are cofirst authors. The order of the co-first authors was determined based on the scope of their contribution to the project and their time of entry into the project.

\section{Acknowledgments}

The authors thank Li Wang, Curtis Olsen, Kathy Zimmerman, Tenley Rawlings, and Xia Liu for technical assistance. Mice harboring floxed alleles for Akt1 were generated by Morris J. Birnbaum's laboratory (University of Pennsylvania, Philadelphia, Pennsylvania, USA) and were provided for this study. RNA-seq data were obtained at the Genomics Division of the Iowa Institute of Human Genetics, which is supported, in part, by the University of Iowa Carver College of Medicine. This work was supported by NIH grants R01DK092065, R01HL070070, R01HL108379, and U01HL087947 to EDA, who is an established investigator of the American Heart Association; R01HL127764 and R01 HL112413 to EDA and YKX; R01DK098655 to MFW; AHA HF 16SFRN29020000 to EDA and SGD; R01HL135121-01, R01HL132067, and Nora Eccles Treadwell Foundation Grant to SGD; and a NIH grant OD019941 to RMW and the Cardiovascular Phenotype Core at the University of Iowa. CR was supported by a postdoctoral fellowship from the German Research Foundation (DFG), ETW by a NIH training grant (5T32HL007638), ARW by an advanced postdoctoral fellowship from the JDRF (10-2009-672) and K99 HL111322 and R01 HL133011 from the NIH, and KMP by the Coordenação de Aperfeiçoamento de Pessoal de Nível Superior (CAPES, Brazil). MR was supported by fellowships from Maastricht University and the Netherlands Heart Foundation (NHF). The graphical abstract was produced using templates from Servier Medical Art (https://smart.servier.com).

Address correspondence to: E. Dale Abel, FOEDRC and Division of Endocrinology and Metabolism, Carver College of Medicine University of Iowa, 4312 PBDB, 169 Newton Road, Iowa City, Iowa 52242, USA. Phone: 319.353.3050; Email: DRCAdmin@uiowa.edu.

1. Heidenreich PA, et al. Forecasting the future of cardiovascular disease in the United States: a policy statement from the American Heart Association. Circulation. 2011;123(8):933-944.

2. Cook SA, et al. Abnormal myocardial insulin signalling in type 2 diabetes and left-ventricular dysfunction. Eur Heart $J$. 2010;31(1):100-111

3. Riehle C, Abel ED. Insulin Signaling and Heart Failure. Circ Res. 2016;118(7):1151-1169.

4. Swan JW, et al. Insulin resistance in chronic heart failure: relation to severity and etiology of heart failure. J Am Coll Cardiol. 1997;30(2):527-532

5. Wang Q, et al. Inhibiting Insulin-Mediated $\beta 2$-Adrenergic Receptor Activation Prevents Diabetes-Associated Cardiac Dysfunction. Circulation. 2017;135(1):73-88.

6. Action to Control Cardiovascular Risk in Diabetes Study Group, et al. Effects of intensive glucose lowering in type 2 diabetes. 
N Engl J Med. 2008;358(24):2545-2559.

7. Dokun AO. Lessons learned from glycemia control studies. Curr Diab Rep. 2010;10(2):133-138.

8. Tamemoto $\mathrm{H}$, et al. Insulin resistance and growth retardation in mice lacking insulin receptor substrate-1. Nature. 1994;372(6502):182-186.

9. Withers DJ, et al. Disruption of IRS-2 causes type 2 diabetes in mice. Nature. 1998;391(6670):900-904.

10. Riehle C, et al. Insulin receptor substrates are essential for the bioenergetic and hypertrophic response of the heart to exercise training. Mol Cell Biol. 2014;34(18):3450-3460.

11. Shimizu I, et al. Excessive cardiac insulin signaling exacerbates systolic dysfunction induced by pressure overload in rodents. J Clin Invest. 2010;120(5):1506-1514.

12. Shende $P$, et al. Cardiac raptor ablation impairs adaptive hypertrophy, alters metabolic gene expression, and causes heart failure in mice. Circulation. 2011;123(10):1073-1082.

13. Matsui T, et al. Phenotypic spectrum caused by transgenic overexpression of activated Akt in the heart. J Biol Chem. 2002;277(25):22896-22901.

14. Rainer PP, Kass DA. Old dog, new tricks: novel cardiac targets and stress regulation by protein kinase G. Cardiovasc Res. 2016;111(2):154-162.

15. Gruzdeva O, Uchasova E, Dyleva Y, Belik E, Shurygina E, Barbarash O. Insulin resistance and inflammation markers in myocardial infarction. J Inflamm Res. 2013;6:83-90.

16. Horwich TB, Fonarow GC. Glucose, obesity, metabolic syndrome, and diabetes relevance to incidence of heart failure. $J$ Am Coll Cardiol. 2010;55(4):283-293.

17. Paolisso G, et al. Elevated plasma fatty acid concentrations stimulate the cardiac autonomic nervous system in healthy subjects. Am J Clin Nutr. 2000;72(3):723-730.

18. Roden M. How free fatty acids inhibit glucose utilization in human skeletal muscle. News Physiol Sci. 2004;19:92-96.

19. Wright JJ, et al. Mechanisms for increased myocardial fatty acid utilization following short-term high-fat feeding. Cardiovasc Res. 2009;82(2):351-360

20. Lin SJ, et al. Calorie restriction extends Saccharomyces cerevisiae lifespan by increasing respiration. Nature. 2002;418(6895):344-348.

21. Chen D, Steele AD, Lindquist S, Guarente L. Increase in activity during calorie restriction requires Sirt1. Science. 2005;310(5754):1641.

22. Fontana L, Partridge L, Longo VD. Extending healthy life span--from yeast to humans. Science. 2010;328(5976):321-326.

23. Wessells RJ, Fitzgerald E, Cypser JR, Tatar M, Bodmer R. Insulin regulation of heart function in aging fruit flies. Nat Genet. 2004;36(12):1275-1281.

24. DeBosch B, Sambandam N, Weinheimer C, Courtois M, Muslin AJ. Akt2 regulates cardiac metabolism and cardiomyocyte survival. J Biol Chem. 2006;281(43):32841-32851.

25. DeBosch B, et al. Akt1 is required for physiological cardiac growth. Circulation. 2006;113(17):2097-2104.

26. Hu P, Zhang D, Swenson L, Chakrabarti G, Abel ED, Litwin SE. Minimally invasive aortic banding in mice: effects of altered cardiomyocyte insulin signaling during pressure overload. Am J Physiol Heart Circ Physiol. 2003;285(3):H1261-H1269.

27. Laustsen PG, et al. Essential role of insulin and insulin-like growth factor 1 receptor signaling in cardiac development and function. Mol Cell Biol. 2007;27(5):1649-1664.

28. Riehle C, et al. Insulin receptor substrate signaling suppresses neonatal autophagy in the heart. J Clin Invest. 2013;123(12):5319-5333.

29. Long YC, Cheng Z, Copps KD, White MF. Insulin receptor substrates Irs1 and Irs2 coordinate skeletal muscle growth and metabolism via the Akt and AMPK pathways. Mol Cell Biol. 2011;31(3):430-441.

30. Qi Y, et al. Myocardial loss of IRS1 and IRS2 causes heart failure and is controlled by p38 $\alpha$ MAPK during insulin resistance. Diabetes. 2013;62(11):3887-3900

31. Layland J, Li JM, Shah AM. Role of cyclic GMP-dependent protein kinase in the contractile response to exogenous nitric oxide in rat cardiac myocytes. $J$ Physiol (Lond). 2002;540(Pt 2):457-467.

32. Shah AM, Spurgeon HA, Sollott SJ, Talo A, Lakatta EG. 8-bromo-cGMP reduces the myofilament response to Ca2+ in intact cardiac myocytes. Circ Res. 1994;74(5):970-978.

33. Seddon M, Shah AM, Casadei B. Cardiomyocytes as effectors of nitric oxide signalling. Cardiovasc Res. 2007;75(2):315-326.

34. Mount PF, Kemp BE, Power DA. Regulation of endothelial and myocardial NO synthesis by multi-site eNOS phosphorylation J Mol Cell Cardiol. 2007;42(2):271-279.

35. Lee DI, et al. PDE5A suppression of acute beta-adrenergic activation requires modulation of myocyte beta-3 signaling coupled to PKG-mediated troponin I phosphorylation. Basic Res Cardiol. 2010;105(3):337-347.

36. Inserte J, Garcia-Dorado D. The cGMP/PKG pathway as a common mediator of cardioprotection: translatability and mechanism. Br J Pharmacol. 2015;172(8):1996-2009.

37. Lee DI, et al. Phosphodiesterase 9A controls nitric-oxide-independent cGMP and hypertrophic heart disease. Nature. 2015;519(7544):472-476.

38. Wang TJ, et al. Impact of obesity on plasma natriuretic peptide levels. Circulation. 2004;109(5):594-600.

39. Magnusson M, et al. Low plasma level of atrial natriuretic peptide predicts development of diabetes: the prospective Malmo Diet and Cancer study. J Clin Endocrinol Metab. 2012;97(2):638-645.

40. Uddin S, et al. Interferon-dependent activation of the serine kinase PI 3'-kinase requires engagement of the IRS pathway but not the Stat pathway. Biochem Biophys Res Commun. 2000;270(1):158-162.

41. Argetsinger LS, Hsu GW, Myers MG, Billestrup N, White MF, Carter-Su C. Growth hormone, interferon-gamma, and leukemia inhibitory factor promoted tyrosyl phosphorylation of insulin receptor substrate-1. J Biol Chem. 1995;270(24):14685-14692.

42. Uddin S, Yenush L, Sun XJ, Sweet ME, White MF, Platanias LC. Interferon-alpha engages the insulin receptor substrate-1 to associate with the phosphatidylinositol 3'-kinase. J Biol Chem. 1995;270(27):15938-15941.

43. Laroumanie F, et al. CD4+ T cells promote the transition from hypertrophy to heart failure during chronic pressure overload. Circulation. 2014;129(21):2111-2124. 
44. Fukunaga T, et al. Expression of interferon-gamma and interleukin-4 production in CD4+ T cells in patients with chronic heart failure. Heart Vessels. 2007;22(3):178-183.

45. Yndestad A, et al. Enhanced expression of inflammatory cytokines and activation markers in T-cells from patients with chronic heart failure. Cardiovasc Res. 2003;60(1):141-146.

46. Nevers T, et al. Left Ventricular T-Cell Recruitment Contributes to the Pathogenesis of Heart Failure. Circ Heart Fail. 2015;8(4):776-787.

47. Kallikourdis M, et al. T cell costimulation blockade blunts pressure overload-induced heart failure. Nat Commun. 2017;8:14680.

48. Wan M, et al. Loss of Akt1 in mice increases energy expenditure and protects against diet-induced obesity. Mol Cell Biol. 2012;32(1):96-106.

49. Russwurm M, Mullershausen F, Friebe A, Jäger R, Russwurm C, Koesling D. Design of fluorescence resonance energy transfer (FRET)-based cGMP indicators: a systematic approach. Biochem J. 2007;407(1):69-77.

50. Reddy GR, et al. Illuminating cell signaling with genetically encoded FRET biosensors in adult mouse cardiomyocytes. $J$ Gen Physiol. 2018;150(11):1567-1582. 